\title{
Critical role of IL-21 and T follicular helper cells in hypertension and vascular dysfunction
}

\author{
Bethany L. Dale, ${ }^{1}$ Arvind K. Pandey, ${ }^{2}$ Yuhan Chen, ${ }^{3}$ Charles D. Smart, ${ }^{1}$ Fanny Laroumanie, ${ }^{3}$ \\ Mingfang Ao, ${ }^{3}$ Liang Xiao, ${ }^{3}$ Anna E. Dikalova, ${ }^{3}$ Sergey I. Dikalov, ${ }^{3}$ Fernando Elijovich, ${ }^{3}$ \\ Jason D. Foss, ${ }^{3}$ Natalia R. Barbaro, ${ }^{3}$ Justin P. Van Beusecum, ${ }^{3}$ Serpil M. Deger, ${ }^{4}$ Aseel Alsouqi, ${ }^{4}$ \\ Hana A. Itani, ${ }^{3}$ Allison E. Norlander, ${ }^{1}$ Matthew R. Alexander, ${ }^{2}$ Shilin Zhao, ${ }^{5}$ T. Alp Ikizler, ${ }^{4}$ \\ Holly M. Scott Algood, 6,7,8 and Meena S. Madhur, ${ }^{1,2,3,7}$ \\ 'Department of Molecular Physiology and Biophysics, Vanderbilt University, Nashville, Tennessee, USA. ${ }^{2}$ Department of \\ Medicine, Division of Cardiovascular Medicine, ${ }^{3}$ Department of Medicine, Division of Clinical Pharmacology, ${ }^{4}$ Department \\ of Medicine, Division of Nephrology, ${ }^{5}$ Department of Biostatistics, and ${ }^{6}$ Department of Medicine, Division of Infectious \\ Disease, Vanderbilt University Medical Center (VUMC), Nashville, Tennessee, USA. ${ }^{7}$ Vanderbilt Institute for Infection, \\ Immunology, and Inflammation, Nashville, Tennessee, USA. ${ }^{8}$ Vanderbilt Digestive Diseases Research Center, Nashville, \\ Tennessee, USA.
}

T and B cells have been implicated in hypertension, but the mechanisms by which they produce a coordinated response is unknown. T follicular helper (Tfh) cells that produce IL-21 promote germinal center (CC) B cell responses, leading to Ig production. Here we investigate the role of IL-21 and Tfh cells in hypertension. In response to angiotensin II-induced (Ang II-induced) hypertension, T cell IL-21 production was increased, and II21/- mice developed blunted hypertension, attenuated vascular end-organ damage, and decreased IL-17A and IFN- $\gamma$ production. Tfh-like cells and CC B cells accumulated in the aorta, and plasma IgG1 was increased in hypertensive WT but not II21/- mice. Furthermore, Tfh cell-deficient mice developed blunted hypertension and vascular hypertrophy in response to Ang II infusion. Importantly, IL-21 neutralization reduced BP and reversed endothelial dysfunction and vascular inflammation. Moreover, recombinant IL-21 impaired endotheliumdependent relaxation ex vivo and decreased NO production from cultured endothelial cells. Finally, we show in humans that peripheral blood T cell production of IL-21 correlated with systolic BP and IL-17A production. These data suggest that IL-21 may be a novel therapeutic target for the treatment of hypertension and its micro- and macrovascular complications.

Conflict of interest: TAl is a consultant for Fresenius Kabi, Corvidia, and Nestle, and MSM holds a patent on monocyte biomarkers for hypertension (PCT/US2018/029482)

Copyright: () 2019 American Society for Clinical Investigation

Submitted: April 1, 2019

Accepted: April 17, 2019

Published: June 6, 2019.

Reference information: /CI Insight. 2019;4(11):e129278. https://doi. org/10.1172/ji.insight.129278.

\section{Introduction}

Hypertension affects nearly one-half of all adults in the United States (1). As a key contributor to vascular disease, chronic kidney disease, and heart failure, hypertension is the leading risk factor for global mortality (2). Unfortunately, nearly $50 \%$ of people with hypertension have uncontrolled BP despite current pharmacological therapies, highlighting the need for a better understanding of the pathophysiology of this disease and identification of novel therapeutic targets (3).

A potential role for immune cells and elevated serum Igs in hypertension was noted more than 50 years ago $(4,5)$, but we are still far from understanding the key immune cell subsets and pathways that lead to hypertension and hypertensive end-organ damage. In 2007, Guzik et al. demonstrated that mice deficient in $\mathrm{T}$ and $\mathrm{B}$ cells develop blunted Ang II-induced hypertension, with the hypertensive response restored by adoptive transfer of T cells (6). Our group and others demonstrated pathogenic effects of specific T cell subsets, most notably IL-17A-producing Th17 cells and IFN- $\gamma$-producing Th1/Tc1 cells, and protective effects of Tregs in hypertension (7). IL-17A and IFN- $\gamma$ induce vascular dysfunction, glomerular injury, and renal salt and water reabsorption $(7,8)$. In 2015, Chan et al. demonstrated that pharmacological or genetic depletion of B cells protects against experimental hypertension (9). The specific $\mathrm{T}$ helper subset that provides help to B cells is T follicular helper (Tfh) cells that produce IL-21, and yet the role of IL-21 and Tfh cells in hypertension is unknown. Importantly, in addition to promoting Th17 and Th1 cells, IL-21 from Tfh cells 
A

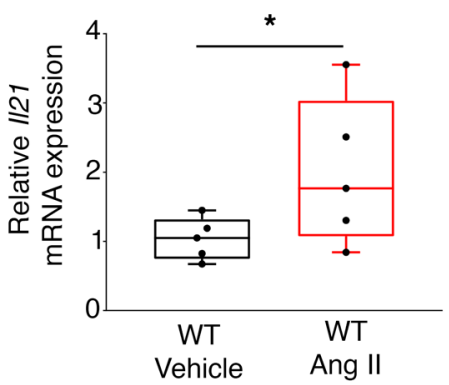

D

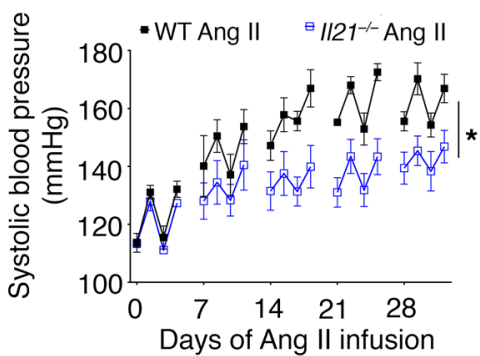

B
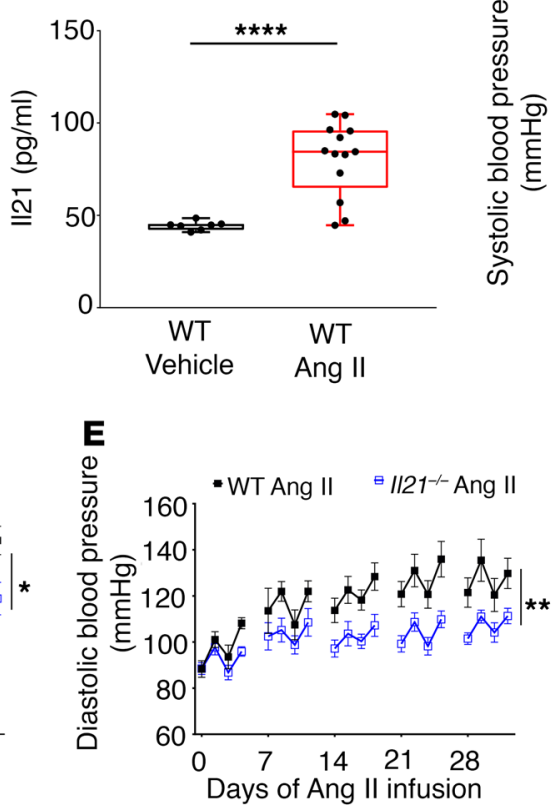

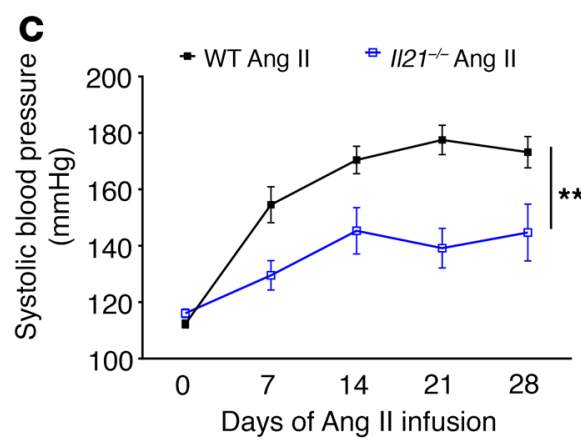

$\mathbf{F}$

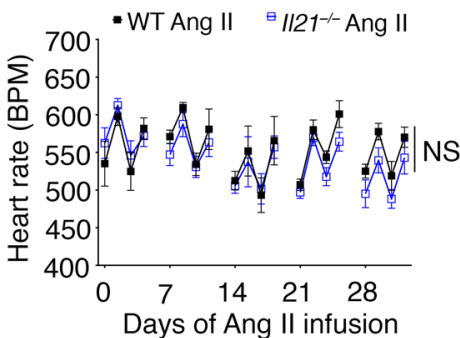

Figure 1. Hypertension is associated with increased CD4 ${ }^{+}$T cell production of IL-21, and IL-21 deficiency blunts the hypertensive response to Ang II infusion. (A) Relative $1 / 21$ mRNA expression by qRT-PCR from splenic CD4+ T cells cultured for 72 hours with anti-CD3/anti-CD28-coated plates ( $n=$ 5). (B) IL-21 protein was quantified in culture supernatants by ELISA $(n=7-13)$. (C) Systolic BP was measured by tail-cuff weekly over 28 days of Ang II infusion in WT and $/ 121^{-/-}$mice $(n=8-9)$. (D) Systolic BP, (E) diastolic BP, and (F) heart rate were measured invasively weekly using carotid radiotelemetry over 28 days of Ang II infusion in WT and $I / 21^{-/-}$mice $(n=5-8)$. Data are expressed as box-and-whisker plots $(\mathbf{A}$ and $\mathbf{B})$ or mean \pm SEM $(\mathbf{C}-\mathbf{F}) ;{ }^{*} P<$ $0.05,{ }^{* *} P<0.01,{ }^{* * *} P<0.0001$ by Student's $t$ test (A and B) or 2-way ANOVA with repeated measures (C-F).

drives a germinal center (GC) reaction in secondary or tertiary lymphoid organs, resulting in GC B cell Ig class switching (e.g., IgM $\rightarrow \operatorname{IgG}$ ) and high-affinity antibody production (10-13). Whether hypertension is associated with a GC response is not known.

Here we report the finding that hypertension is associated with increased Tfh and GC B cells in the aorta and increased GC B cells in secondary lymphoid organs along with increased plasma IgG1. Using genetic and/or pharmacological inhibition of IL-21 and Tfh cells, we demonstrate what we believe to be a previously undefined role for IL-21 in hypertension pathogenesis and vascular dysfunction, suggesting that IL-21 and Tfh cells may serve as novel therapeutic targets for this disease.

\section{Results}

Hypertension is associated with increased $C D 4^{+} T$ cell production of IL-21, and IL-21 deficiency attenuates the rise in $B P$ in response to hypertensive stimuli. To determine the role of IL-21 in hypertension, we first isolated splenic $\mathrm{CD}^{+} \mathrm{T}$ cells from C57BL/6J WT mice infused with vehicle or Ang II for 4 weeks and cultured them in the presence of anti-CD3 and anti-CD28 antibodies for 72 hours. We found that $C D 4^{+} \mathrm{T}$ cells from hypertensive mice showed increased II21 mRNA expression (Figure 1A) and increased IL-21 secretion (Figure 1B) We then investigated the effect of IL-21 deficiency on Ang II-induced hypertension. Following 4 weeks of

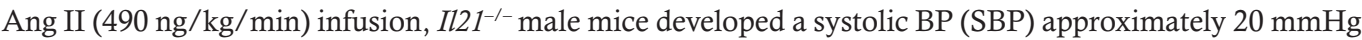
lower than that in WT animals by tail cuff (Figure 1C) and invasive radio telemetry (Figure 1D). Diastolic $\mathrm{BP}$ was similarly reduced in $I l 21^{-/-}$mice compared with WT controls, with no change in heart rate (Figure $1, \mathrm{E}$ and $\mathrm{F}$ ). To determine whether there are sex differences in the effect of IL-21 on hypertension, we studied female mice and found that both the hypertensive response and effect of IL-21 deficiency were similar to those observed in male mice (Supplemental Figure 1A; supplemental material available online with this article; https://doi.org/10.1172/jci.insight.129278DS1). To confirm that the BP protection was not unique to the Ang II model, we investigated the effect of IL-21 deficiency in a salt-sensitive hypertension model characterized by uninephrectomy, implantation of a deoxycorticosterone acetate (DOCA) pellet, and 1\% $\mathrm{NaCl}$ in the drinking water and observed a similar 20-mmHg reduction in SBP (Supplemental Figure 1B). All further studies were conducted in male mice using the Ang II hypertension model. 
Loss of IL-21 protects against Ang II-induced vascular remodeling and endothelial dysfunction. Hypertension is associated with increased aortic collagen deposition, vascular smooth muscle cell hypertrophy, and microvascular endothelial dysfunction. To determine the effect of IL-21 deficiency on vascular remodeling and endothelial function, WT and $I l 21^{-/-}$mice were infused with vehicle or Ang II for 4 weeks. Importantly, thoracic aortas of $\mathrm{Il21^{-/- }}$ mice exhibited significantly less collagen deposition and reduced medial hypertrophy (representative aortic cross sections, Figure 2, A and B; quantification, Figure 2C). Resistance artery endothelial function was assessed by measuring endothelium-dependent and -independent relaxation of third-order mesenteric arterioles. A modest baseline impairment in endothelium-dependent relaxation in response to acetylcholine (Ach) was present in $I l 21^{-/-}$mesenteric arterioles, but the key finding was that the $I I 21^{-1-}$ mice were completely protected from further endothelial dysfunction in response to Ang II infusion, while WT vessels exhibited a severe impairment in endothelium-dependent relaxation in response to Ang II. There was no effect of Ang II or IL-21 deficiency on endothelium-independent relaxation in response to sodium nitroprusside (SNP) (Figure 2D). Since vascular reactivity can be mediated by alterations in superoxide levels, we measured superoxide production in isolated mesenteric arterioles. Interestingly, $I l 21^{-1-}$ mice exhibited increased superoxide levels at baseline, consistent with their baseline impairment in vascular reactivity, but no further increase in response to Ang II infusion. In contrast, WT mice exhibited a marked upregulation of superoxide production in response to Ang II-induced hypertension (Figure 2E).

IL-21 deficiency blunts early vascular infiltration of NKT cells and macrophages. We previously showed that during the initial phase of Ang II-induced hypertension (day 7), there is an increase in total aortic leukocytes composed primarily of innate immune cells such as $\mathrm{F} 4 / 80^{+}$monocytes/macrophages (14). Here we quantified total leukocytes $\left(\mathrm{CD} 45^{+}\right)$, NKT cells $\left(\mathrm{CD}^{+} \mathrm{NK} 1.1^{+}\right)$, macrophages $\left(\mathrm{F} 4 / 80^{+} \mathrm{Ly}_{6 \mathrm{G}}^{-}\right)$, and neutrophils $\left(\mathrm{F} 4 / 80^{-} \mathrm{Ly}_{6 \mathrm{G}}{ }^{+}\right)$in the aorta of WT and $\mathrm{Il21}^{-/-}$mice following 7 days of vehicle or Ang II infusion. Flow cytometry gating strategy and fluorescence minus one (FMO) controls are shown in Supplemental Figure 2. Macrophages and neutrophils were gated from $\mathrm{CD} 11 \mathrm{~b}^{+}$cells, and NKT cells were gated from $\mathrm{CD}^{+}$cells (Supplemental Figure 2). Representative biaxial flow cytometry dot plots are shown for each group (Figure 3A). We found that Ang II-induced hypertension was associated with an early increase in total leukocytes, NKT cells, and macrophages, and this increase was abrogated in $I l 21^{-{ }^{-}}$mice (Figure $3 \mathrm{~B}$ ). Neutrophils were not increased by Ang II infusion or affected by IL-21 deficiency (Figure 3B). Thus, IL-21 deficiency protects from early innate immune cell infiltration into the aorta.

Hypertension induces GC-like cells in the vasculature and increased splenic T cell cytokine production in an IL-21-dependent manner. Peripheral GC-like cells have been found in inflamed tissues in autoimmune conditions such as multiple sclerosis, rheumatoid arthritis, and lupus nephritis (15-17). Since IL-21 is a key driver of the GC reaction and produced predominantly by Tfh or peripheral helper $\mathrm{T}(\mathrm{Tph})$ cells (which do not express the lymphoid follicle homing chemokine receptor CXCR5 but still retain PD-1) (16), we determined whether the numbers of these cells and GC B cells were altered in the aorta during experimental hypertension. WT and $I l 21^{-I_{-}}$mice were infused with vehicle or Ang II for 4 weeks. Flow cytometry gating strategy and FMO controls are shown in Supplemental Figures 3 and 4 . Total leukocytes, CD4 ${ }^{+} \mathrm{T}$ cells, and CD19+B220 $\mathrm{B}$ cells increased in WT mice in response to 4 weeks of Ang II infusion, and this increase was blunted in $I l 21^{-/-}$mice (Supplemental Figures 3 and 4). Tfh cells and Tph cells were gated from CD4 ${ }^{+} \mathrm{T}$ cells, and GC B cells were gated from total B cells that were also $\mathrm{IgD}^{-}$and $\mathrm{CD} 138^{-}$. Representative biaxial flow cytometry dot plots for Tph cells (PD-1 $\left.{ }^{+} \mathrm{CXCR} 5^{-}\right)$, Tfh cells $\left(\mathrm{PD}-1^{+} \mathrm{CXCR} 5^{+}\right.$), and GC B cells $\left(\mathrm{Fas}^{+} \mathrm{GL}^{+}\right)$are shown for each group (Figure $4, \mathrm{~A}$ and $\mathrm{B}$ ). We found that Tph, Tfh, and GC B cells accumulated in the aorta of WT but not $I l 21^{-/-}$animals in response to Ang II-induced hypertension (Figure 4C). We previously showed that hypertension is associated with increased $\mathrm{T}$ cell production of the proinflammatory cytokines IL-17A and IFN- $\gamma(18,19)$. IL-21 can potentially modulate the balance of Th17, Th1, and Treg subsets in disease $(11,20)$. Therefore, we investigated the effect of IL-21 deficiency on T cell production of IL-17A, IFN- $\gamma$, and IL-10 in hypertension. Consistent with our prior results, we detected an increase in $\mathrm{CD}^{+} \mathrm{T}$ cell production of IL-17A and $\mathrm{CD} 8^{+} \mathrm{T}$ cell production of IFN- $\gamma$ in splenic $\mathrm{T}$ cells cultured from hypertensive versus normotensive mice, and these hypertension-induced changes were completely abrogated in $I l 21^{-/-}$mice. Neither Ang II nor IL-21 deficiency affected CD4+ $\mathrm{T}$ cell production of IL-10 (Figure 4D). Thus, hypertension is associated with increased aortic accumulation of Tph, Tfh, and GC B cells and increased splenic T cell production of IL-17A and IFN- $\gamma$ in an IL-21-dependent manner.

Hypertension induces an IL-21-dependent GC response, tertiary lymphoid development, and increased IgG production. The primary effector function of Tfh cells is to stimulate GC B cells in secondary and tertiary lymphoid organs to undergo cycles of proliferation and selection, leading to the production of 
A

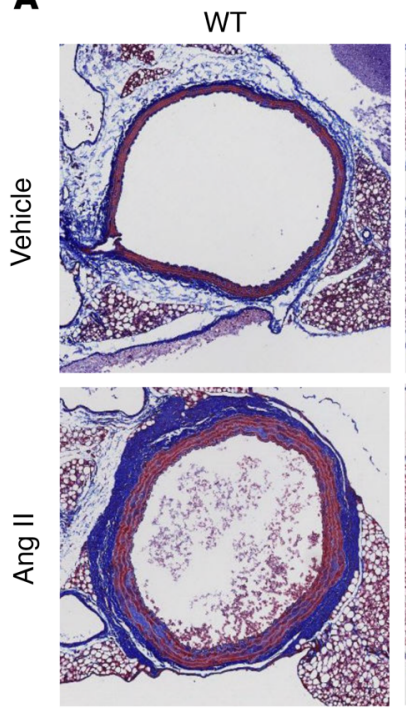

C

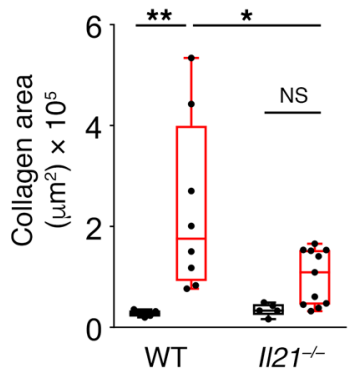

$1121^{-1-}$
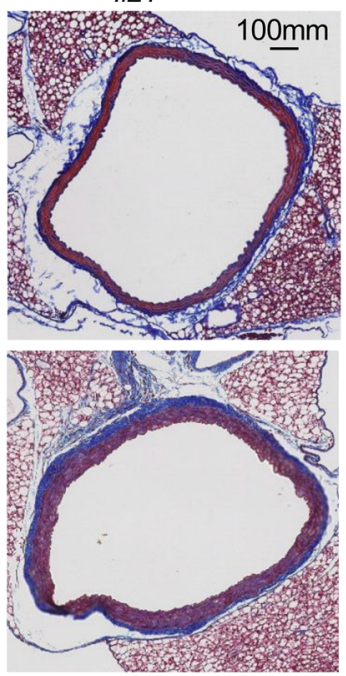

$\square$ Ang II
B

$\frac{0}{0}$

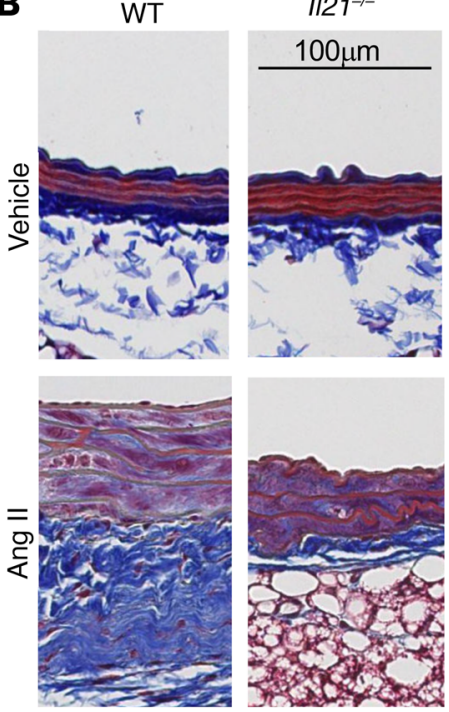

D

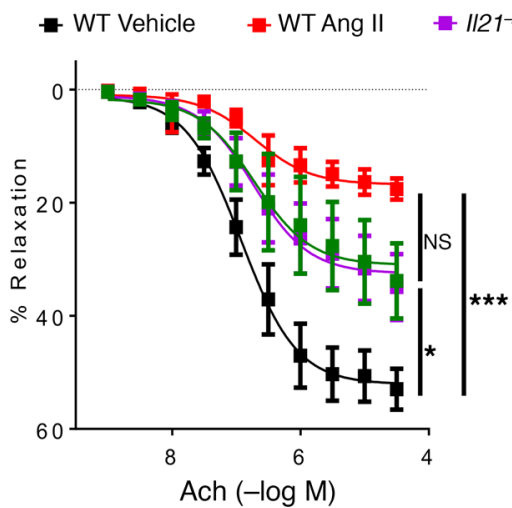

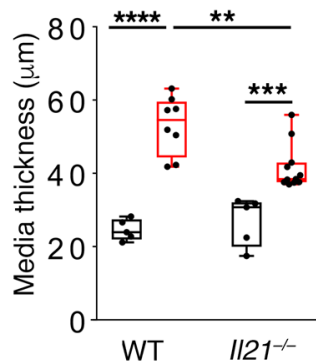

E

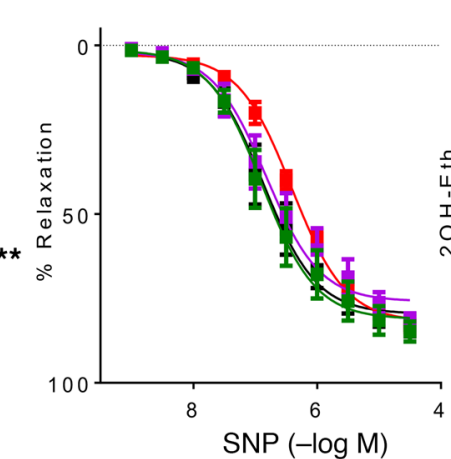

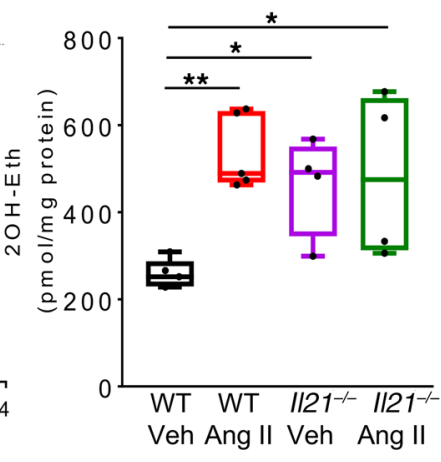

Figure 2. Loss of IL-21 protects against Ang II-induced vascular remodeling and endothelial dysfunction. WT and $1121^{-1-}$ mice were infused with Ang $\|$ or vehicle for 28 days. Representative images of (A) bright-field aortic collagen deposition and (B) media thickness by Masson's trichrome blue staining. Scale bars: $100 \mathrm{~mm}$ (A); 100 $\mu \mathrm{m}$ (B). (C) Quantification of aortic collagen deposition area and media thickness $(n=5-11)$. (D) Endothelium-dependent relaxation in response to increasing doses of acetylcholine (Ach) (left) and endothelium-independent relaxation in response to increasing doses of sodium nitroprusside (SNP) (right) were measured in isolated mesenteric arterioles $(n=6-8)$. (E) Superoxide levels from mesenteric arterioles as measured by quantification of 2-hydroxyethidium (2OH-Eth) $(n=4-5)$. Data are expressed as box-and-whisker plots (C and $\mathbf{E})$ or mean \pm SEM (D); ${ }^{*} P<0.05,{ }^{* *} P<0.01,{ }^{* *} P<0.001,{ }^{* * *} P<0.0001$ by 2 -way ANOVA (C-E). 


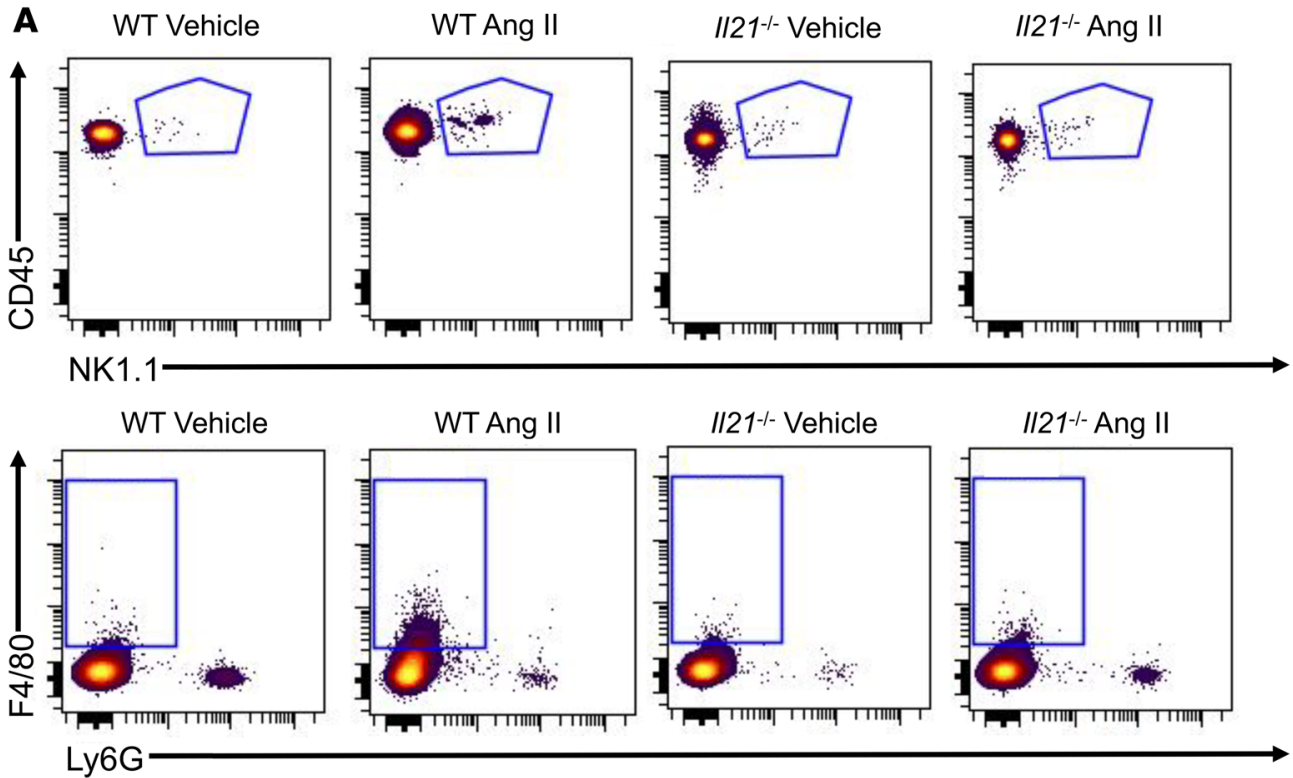

B $\square$ Vehicle $\square$ Ang II
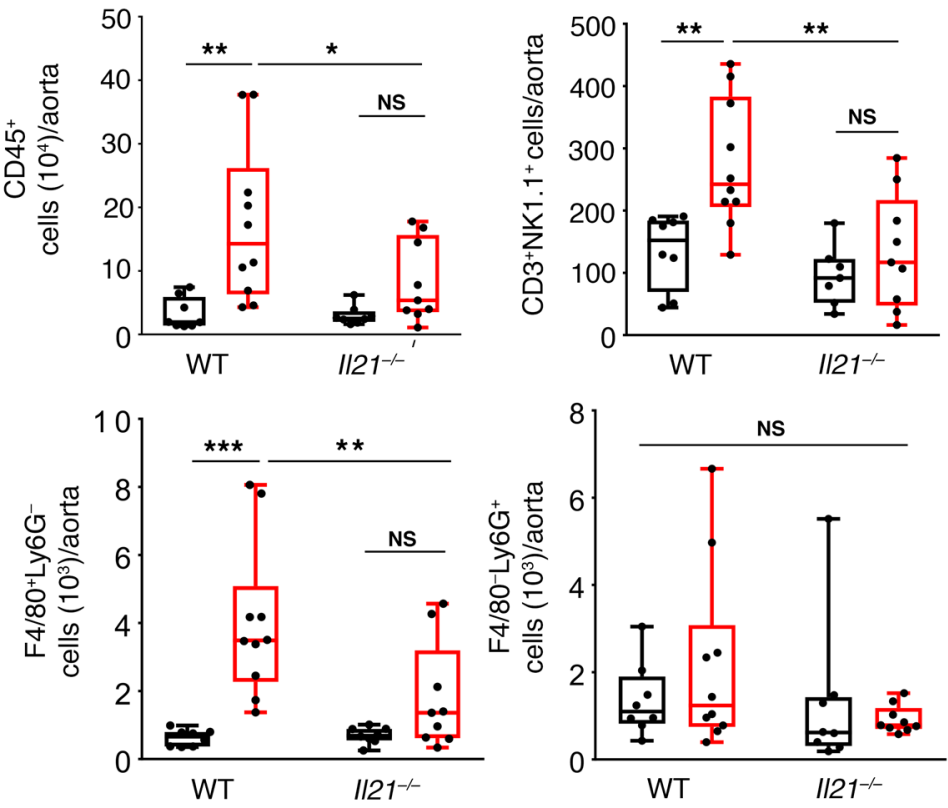

Figure 3. IL-21 deficiency blunts early vascular infiltration of NKT cells and macrophages. (A) Representative flow

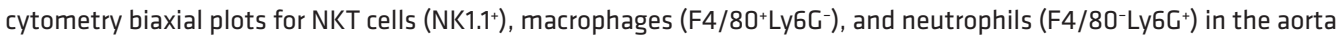
of WT and $1 / 21^{-1-}$ mice infused with Ang II or vehicle for 7 days. (B) Summary quantification of leukocytes (CD45+ cells), NKT cells (CD3+NK1.1+ cells), macrophages (F4/80+ $\left.\mathrm{Ly6G}^{-}\right)$, and neutrophils (F4/80- $\left.\mathrm{LyGG}^{+}\right)(n=7-10)$. Data are expressed as box-and-whisker plots; ${ }^{*} P<0.05,{ }^{* *} P<0.01,{ }^{* *} P<0.001$ by 2 -way ANOVA.

class-switched and/or high-affinity antibodies and long-lived plasma cells (21). Although B cells have recently been implicated in the pathogenesis of hypertension (9), it is not known whether hypertension is associated with a GC reaction as observed in the setting of infection, vaccination, and some autoimmune diseases (22). Due to their proximity to mesenteric resistance vessels in which IL-21 deficiency was shown to play a protective role, we isolated mesenteric lymph nodes from WT and $I l 21^{-/-}$mice infused with vehicle or Ang II for 4 weeks and quantified Tfh and GC B cells. Tfh cells were expressed as percent of total $\mathrm{CD} 4^{+} \mathrm{T}$ cells, and GC B cells were expressed as percent of total B cells. Ang II infusion did not alter the percentage of Tfh cells in mesenteric lymph nodes of WT mice, but Tfh cells were reduced 
A

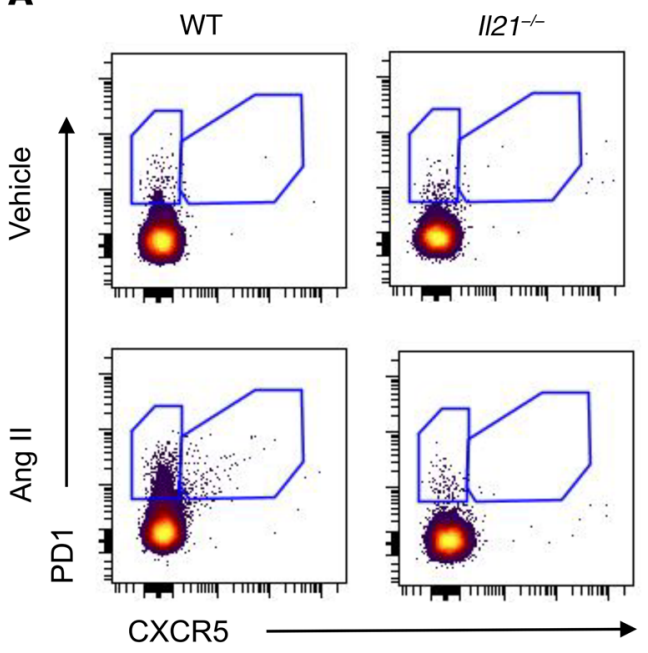

B

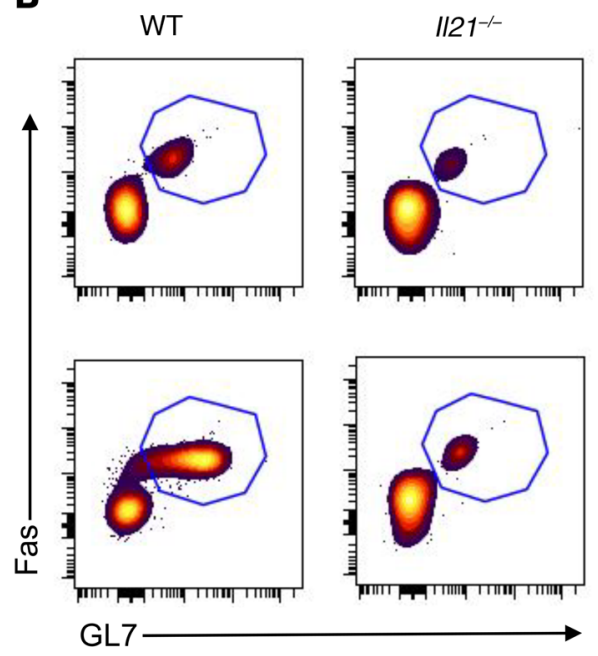

C

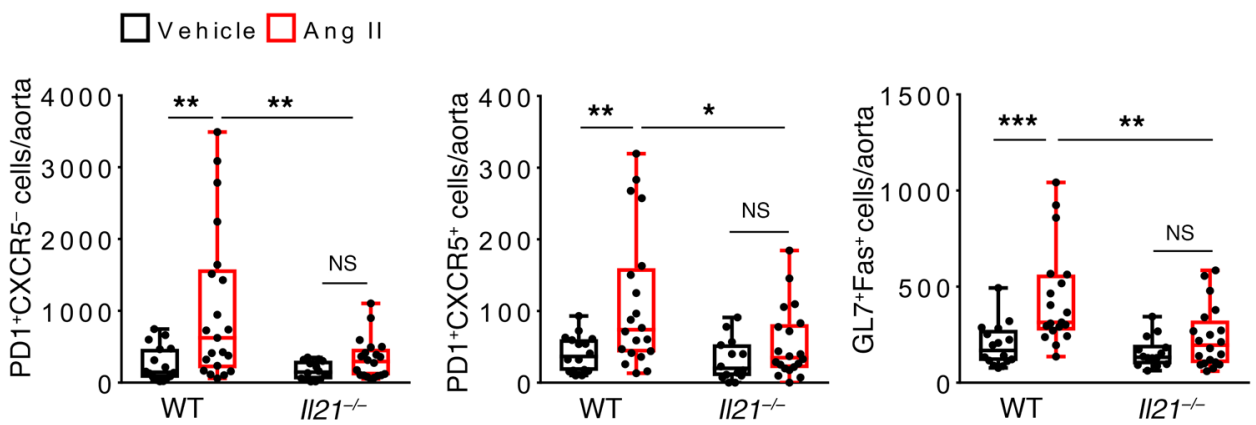

D

$\square$ Vehicle $\square$ Ang II
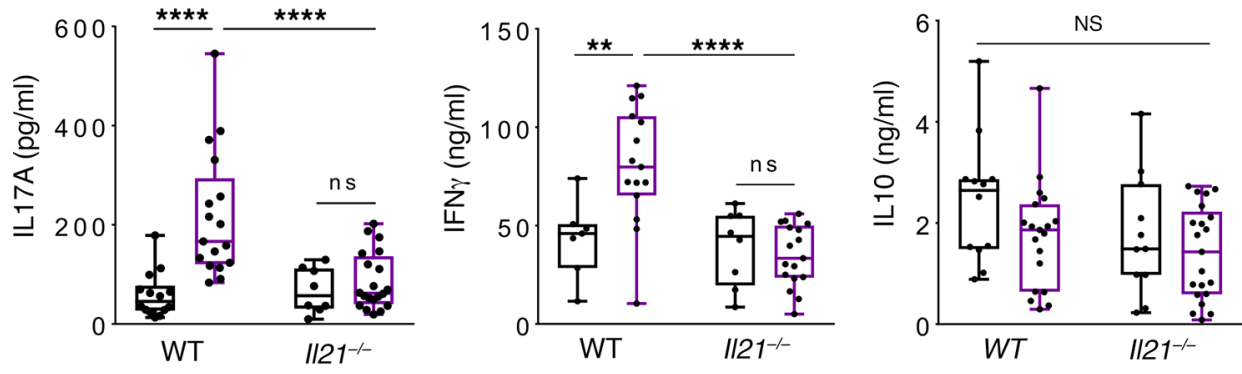

Figure 4. Hypertension induces peripheral T helper, $T$ follicular helper, and GC B cells in the aorta in an IL-21dependent manner. (A) Representative flow cytometry biaxial plots for peripheral T helper (Tph; PD- $\left.1^{+} C X C R 5^{-}\right), T$ follicular helper (Tfh; PD- $1^{+}$CXCR5 $5^{+}$, and (B) GC B cells (CL7+Fas ${ }^{+}$) in the aorta of WT and $/ 121^{-1-}$ mice infused with Ang II or vehicle for 28 days. (C) Summary quantification of Tph, Tfh, and GC B cells ( $n=14-21)$. (D) Splenic CD4+ T cell production of IL-17A and IL-10 and CD8+ T cell production of IFN- $\gamma$ from WT and $/ / 21^{-/-}$mice infused with Ang II or vehicle for 28 days quantified by ELISA $(n=7-21)$. Data are expressed as box-and-whisker plots; ${ }^{*} P<0.05,{ }^{*} P<$ $0.01,{ }^{* *} P<0.001,{ }^{* * *} P<0.0001$ by 2 -way ANOVA.

both at baseline (vehicle infusion) and after Ang II infusion in $I l 21^{-1-}$ mice (Figure 5A). Interestingly, the percentage of GC B cells increased in mesenteric lymph nodes of WT mice in response to Ang

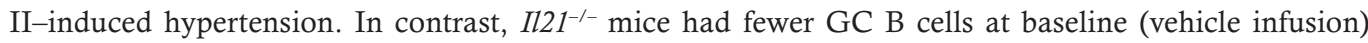
and exhibited no increase in these cells after Ang II infusion (Figure 5B). Of note, neither hypertension nor IL-21 deficiency was associated with changes in overall immune cell numbers or composition in the mesenteric lymph nodes, as total $\mathrm{CD} 45^{+}$leukocytes, percent $\mathrm{CD} 4^{+} \mathrm{T}$ cells, and percent total $\mathrm{B}$ cells were unchanged in all 4 groups (Supplemental Figure 5A). We then investigated follicular architecture and GCs in the spleens of WT and Il21 $1^{-/-}$mice following 4 weeks of vehicle or Ang II infusion. 
A

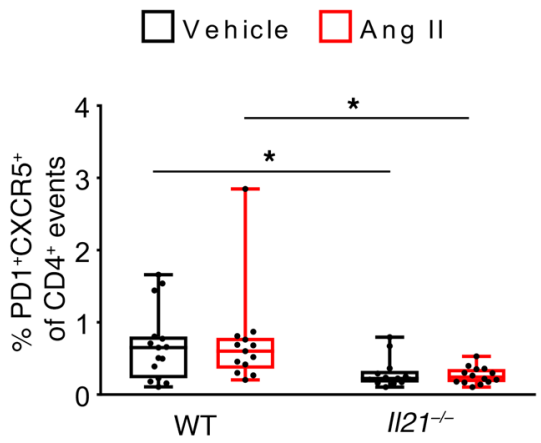

C

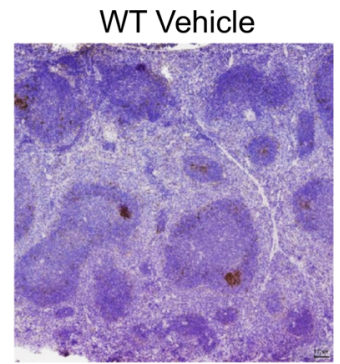

D

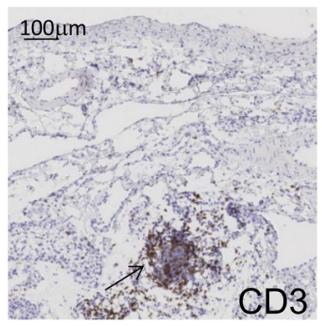

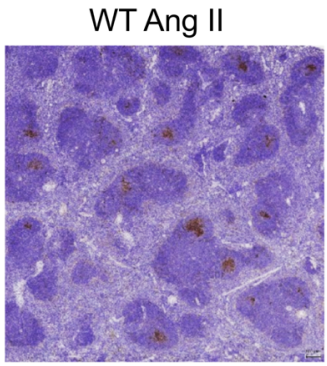

B $\square$ vehicle $\square$ Ang II
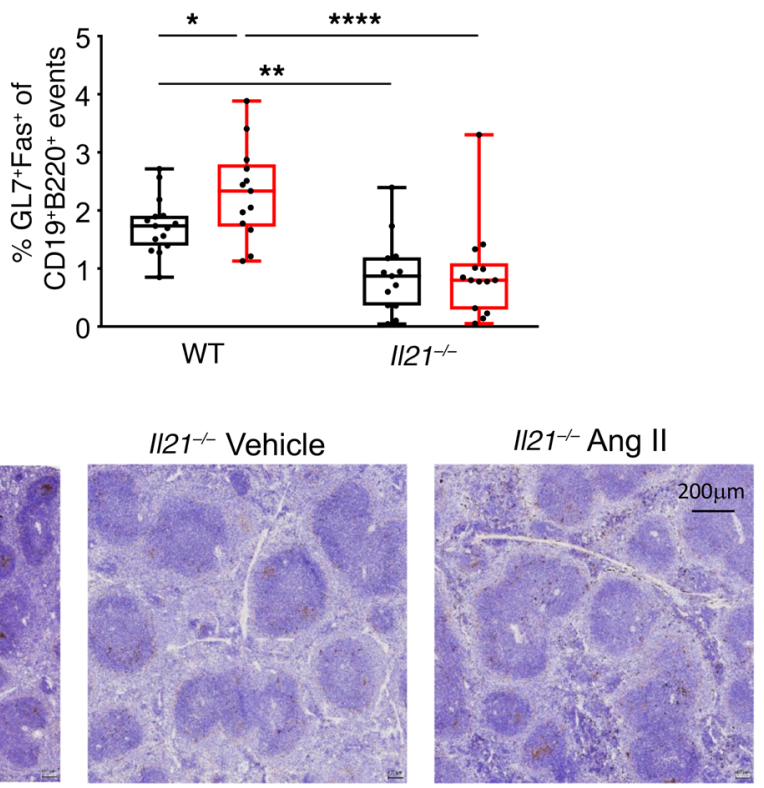

WT Ang II
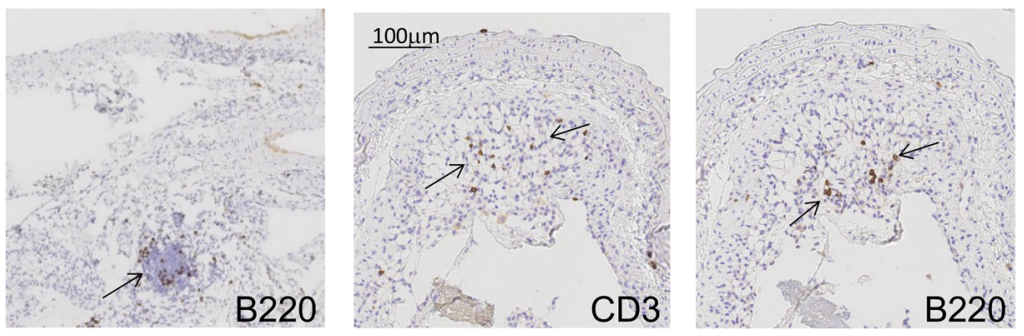

E

Vehicle $\square$ Ang II
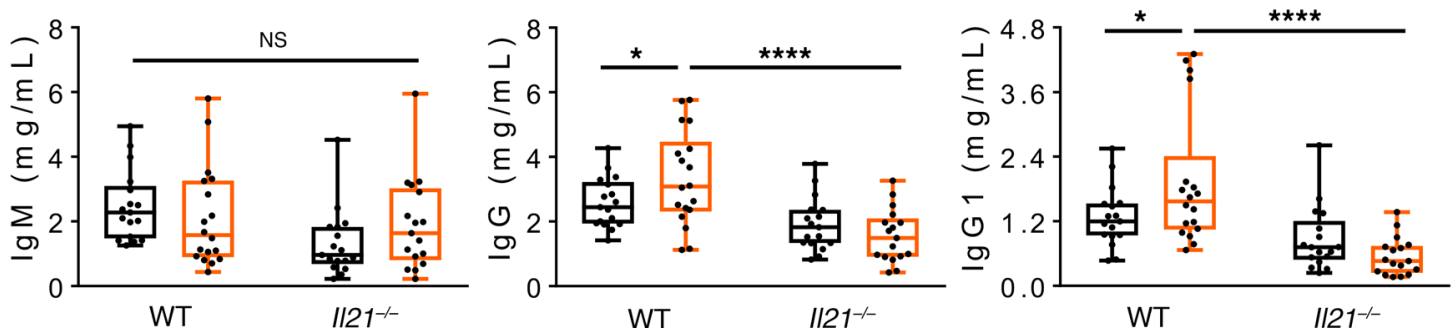

Figure 5. Hypertension induces an IL-21-dependent GC response, tertiary lymphoid development, and increased IgG production. WT and II27/- mice were infused with Ang II or vehicle for 28 days. (A) Percent Tfh cells (PD- $\left.1^{+} C X C R 5^{+}\right)$of $C D 4^{+}$T cells and (B) percent GC B cells (CL7 $7^{+}$Fas $\left.{ }^{+}\right)$of total CD19+B220 B cells from mesenteric lymph nodes $(n=13-15)$. (C) Bright-field images of splenic cross sections stained for the GC B cell marker PNA (representative of $n=4-7)$. (D) Example bright-field images of aortic sections stained for CD3 (T cells) and B220 (B cells). Scale bars: $200 \mu \mathrm{m}$ (C); $100 \mu \mathrm{m}$ (D). (E) Plasma lg concentration of IgM, IgG, and IgG1 $(n=17-18)$. Data are expressed as box-and-whisker plots; ${ }^{*} P<0.05,{ }^{* *} P<0.01,{ }^{* * *} P<0.0001$ by 2 -way ANOVA.

Interestingly, and consistent with the findings of Chan and colleagues (9), we observed an increase in follicle number and decrease in follicle size in spleens of hypertensive WT mice, and this finding was attenuated in $I l 21^{-/-}$mice. Furthermore, PNA staining, which detects GC B cells, was weaker in $I l 21^{-1-}$ spleens (Figure 5C), consistent with the findings of Linterman et al. (12). To determine whether tertiary lymphoid organs form in hypertensive aortas, we performed whole mount immunohistochemistry of aortas from WT mice infused with Ang II for 4 weeks. We found evidence of $\mathrm{T}$ and B cell clusters resembling tertiary lymphoid organs, as well as sites of unstructured $\mathrm{T}$ and $\mathrm{B}$ cell aggregates (Figure 5D) in hypertensive aortas. We then quantified plasma Igs in WT and $I l 21^{-1-}$ mice infused for 4 weeks with 
A

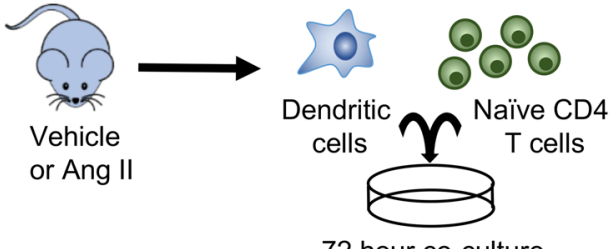

72 hour co-culture

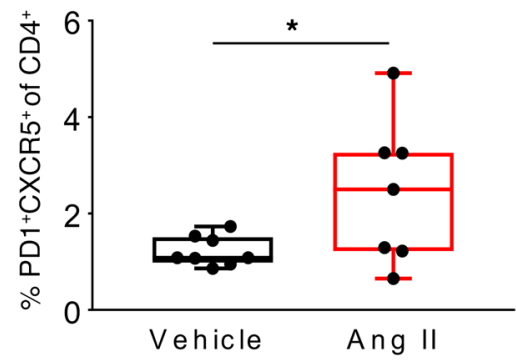

C

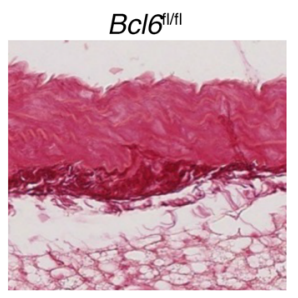

$B c / 6^{f / 1 / 1} T_{g} \mathrm{CD} 4 \mathrm{cre}$

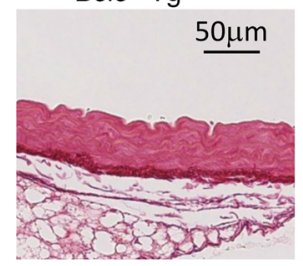

B

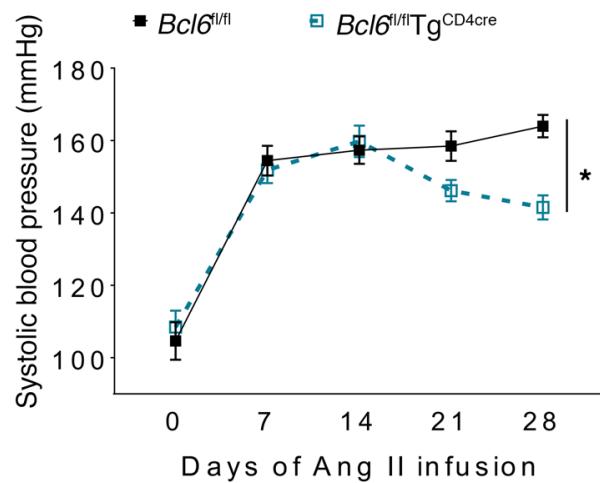

D

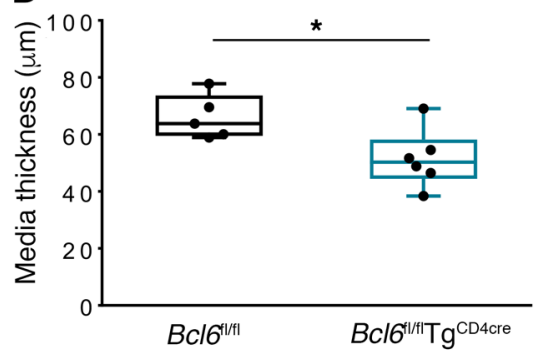

Figure 6. Hypertensive DCs induce Tfh cell polarization, and Tfh cells play a critical role in hypertension.

(A) DCs were isolated from the spleen of WT mice infused with Ang II or vehicle for 14 days and cocultured with splenic naive $\mathrm{CD}^{+} \mathrm{T}$ cells from vehicle-infused WT mice (top). Percent Tfh cells (PD-1+CXCR5 ${ }^{+}$) of CD4 ${ }^{+} T$ cells was determined by flow cytometry ( $n=7-8)$ (bottom). (B) Systolic BP was measured by tail-cuff weekly over 28 days of Ang II infusion in $B c / 6^{f / f l} T g^{\text {cDAcre }}$ and control $B c / 6^{f / f}$ littermates ( $n=9-11)$. (C) Representative images of bright-field aortic wall thickness by Picrosirius red staining. Scale bar: $50 \mu \mathrm{m}$. (D) Quantification of aortic wall thickness $(n=5-6)$.

(E) Summary quantification of total leukocytes (CD45'), T helper (CD4+), B (CD19+B220+), Tph (PD-1+CXCR5 $\left.{ }^{-}\right)$, Tfh (PD-1+CXCR5 ${ }^{+}$), and GC B (GL7+ $\left.{ }^{+} \mathrm{Fs}^{+}\right)$ cells in the aorta from Bcl $6^{\text {flfl }} \mathrm{Tg}^{\text {CD4cre }}$ and control $B c / 6^{f / / f}$ littermates infused with Ang II for 28 days ( $n=9-10$ ). Data are expressed as box-and-whisker plots $(\mathbf{A}, \mathbf{D}$, and $\mathbf{E})$ or mean \pm SEM (B); ${ }^{*} P<0.05$ by Student's $t$-test $(\mathbf{A}, \mathbf{D}$, and $\mathbf{E})$ or 2-way ANOVA with repeated measures $(\mathbf{B})$.
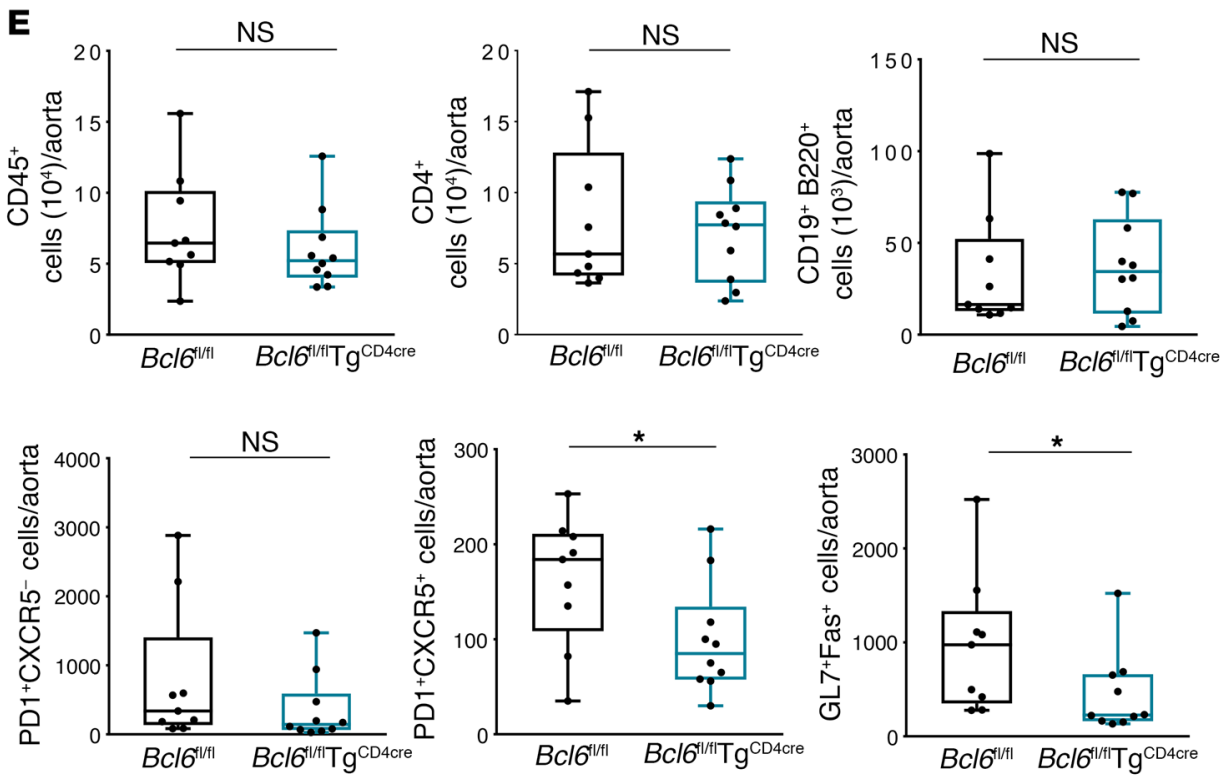

vehicle or Ang II. Ang II-induced hypertension was associated with an increase in total IgG, driven primarily by the IgG1 subclass, in WT but not $I l 21^{-/-}$mice. IgG2a was not significantly increased by Ang II infusion but was higher in Ang II-infused WT versus $I l 21^{1^{--}}$mice. There was no change in the other IgG subclasses or IgM among all 4 groups (Figure 5E and Supplemental Figure 5B). Taken together, these data suggest that hypertension is associated with an IL-21-dependent GC response.

Hypertensive DCs induce Tfh cell polarization, and Tfh cells play a critical role in hypertension. Hypertensive stimuli have been shown to activate DCs, which in turn stimulate T cell proliferation through presentation of isolevuglandin-modified peptides (23). To determine whether hypertensive DCs promote naive $\mathrm{T}$ cells to polarize to Tfh cells, we utilized a coculture assay. Splenic DCs from mice infused with vehicle or Ang II for 
A

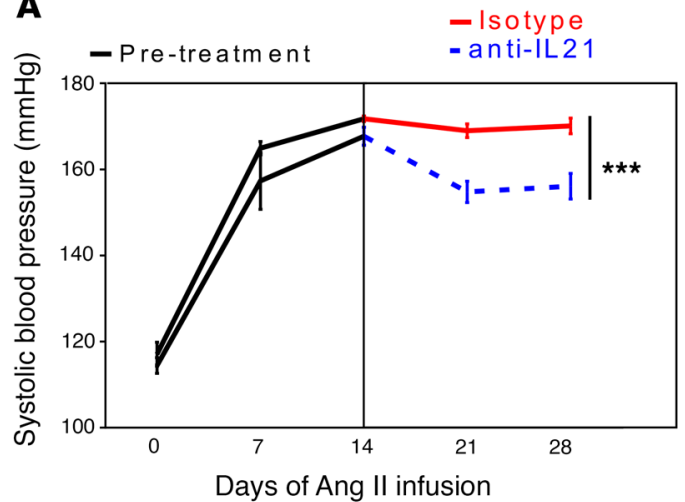

C

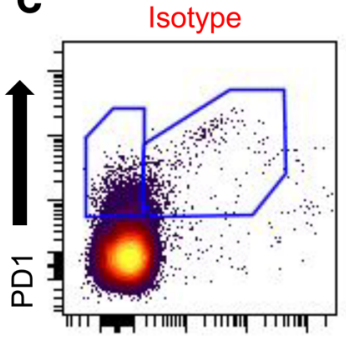

CXCR5
B

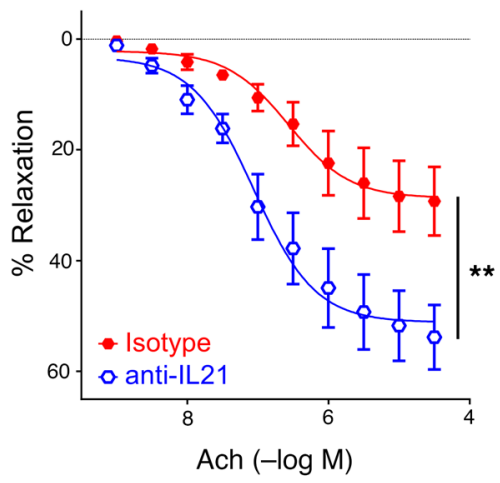

D
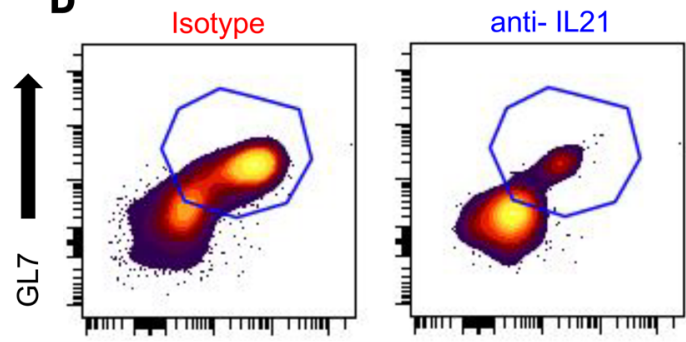

Fas
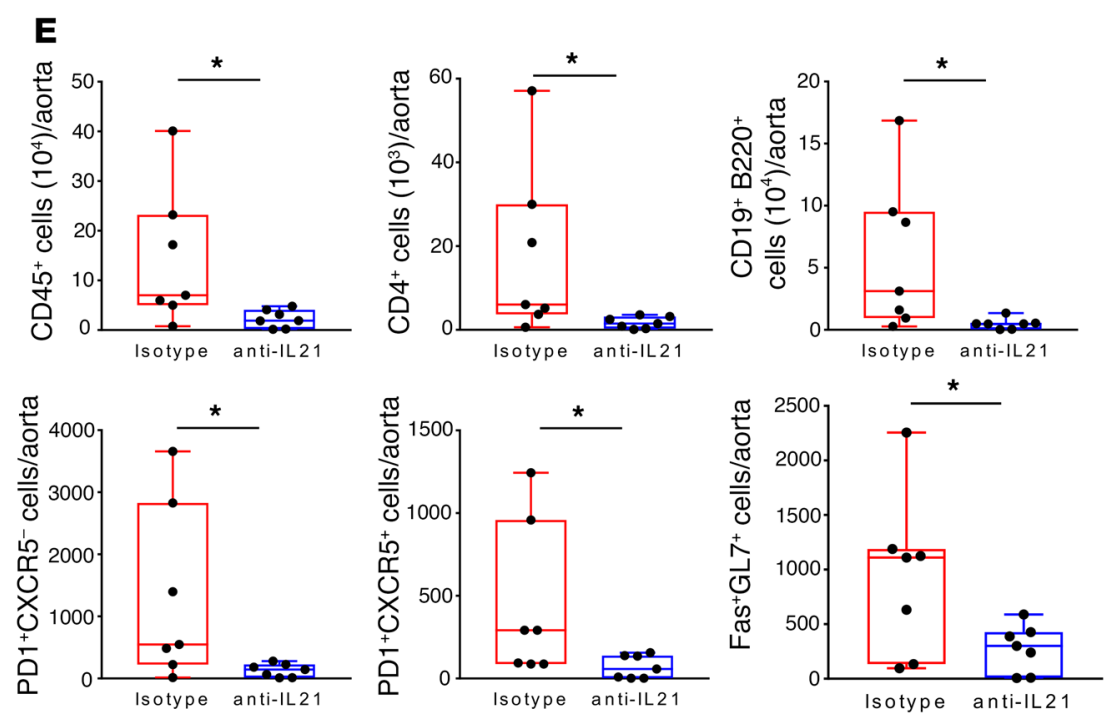

Figure 7. Anti-IL-21 treatment lowers BP and reverses endothelial dysfunction and vascular inflammation. (A) Systolic BP was measured by tail-cuff weekly over 28 days of Ang II infusion. Isotype control or IL-21-neutralizing antibodies were administered twice weekly during the last 2 weeks of Ang II infusion ( $n=7-8)$. (B) Endothelium-dependent relaxation in response to increasing doses of Ach was measured in both groups $(n=7-8)$. Representative flow cytometry biaxial plots for (C) Tph (PD-1+CXCR5-), Tfh (PD-1+CXCR5 $5^{+}$, and (D) GC B (CL7+Fas $)$cells in the aorta from both groups. (E) Summary quantification of total leukocytes (CD45+), T helper (CD4+), B (CD19+B220+), Tph, Tfh, and GC B cells from both groups $(n=7)$. Data are expressed as mean \pm SEM $(\mathbf{A}$ and $\mathbf{B})$ or box-and-whisker plots $(\mathbf{E}) ;{ }^{*} P<0.05$, ${ }^{* *} P<0.01,{ }^{* * *} P<0.001$ by 2 -way ANOVA with repeated measures (A) or Student's $t$ test (B and $\left.\mathbf{E}\right)$.

2 weeks were isolated and cocultured with naive $\mathrm{CD} 4^{+} \mathrm{T}$ cells at a 1:5 ratio for 72 hours (Figure 6A). Hypertensive DCs induced a 2-fold increase in Tfh cell polarization compared with normotensive DCs (Figure 6A). Since BCL6 is an essential transcription factor for the Tfh cell differentiation program, we generated mice with Tfh cell deficiency by crossing floxed BCL6 mice $\left(B c l 6^{\text {B/fff}}\right)$ with mice transgenic for Cre recombinase under the control of the $\mathrm{CD} 4$ promoter $\left(\mathrm{Tg}^{\mathrm{CDAcr}}\right)$. To confirm $\mathrm{T}$ cell deletion of $\mathrm{BCL} 6$, we isolated $\mathrm{CD} 4^{+}$ 
A

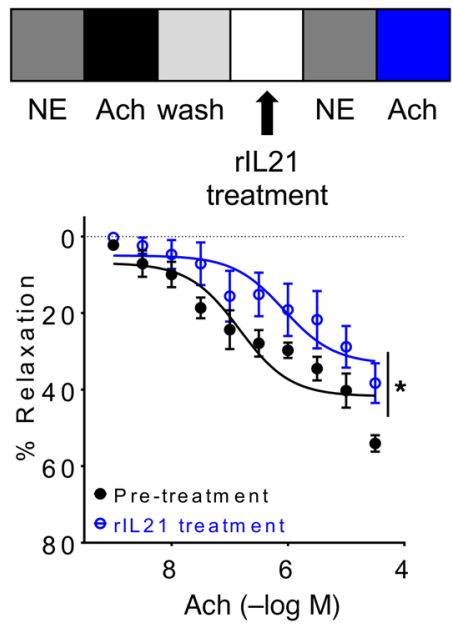

C

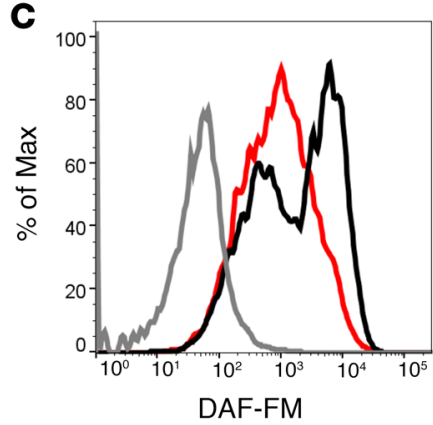

B
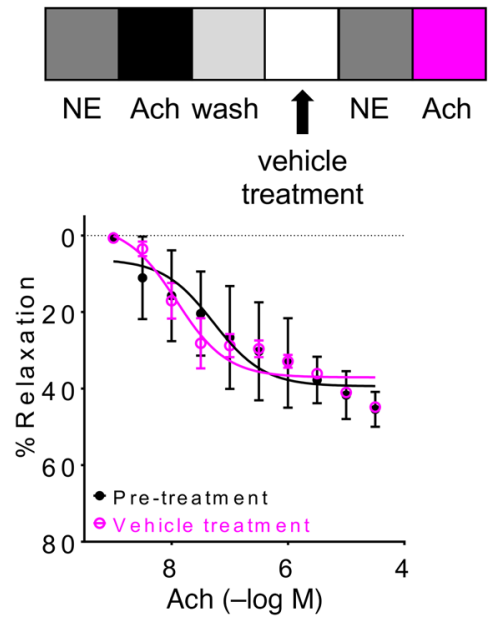

$\square$ Unstained

Vehicle

rIL21

Figure 8. Recombinant IL-21 impairs endothelium-dependent relaxation ex vivo and decreases NO production from cultured human aortic endothelial cells. (A and B) Schematic of ex vivo treatment of WT mesenteric arterioles (top), and endothelium-dependent relaxation in response to increasing doses of acetylcholine (Ach) before and after treatment with recombinant IL-21 (rIL21) $(\mathbf{A}, n=5)$ or vehicle $(\mathbf{B}, n=3)$. NE, norepinephrine. (C) Histogram of 4-amino-5-methylamino-2', $7^{\prime}$-diflurofluorescein diacetate (DAF-FM) fluorescence in cultured human aortic endothelial cells treated with recombinant IL-21 or vehicle (representative of $n=4$ ). Data are expressed as mean \pm SEM (A and $\mathbf{B}) ;{ }^{*} P<0.05$ by paired Student's $t$ test $(\mathbf{A}$ and $\mathbf{B})$.

T cells from the spleen of $B c l 6^{A / f l} \mathrm{Tg}^{\mathrm{CDAcre}}$ mice and Cre-negative $B c l 6^{A / f l}$ littermate controls and demonstrated a significant reduction in $B c l 6$ expression by quantitative real-time PCR (qRT-PCR) in $B c l 6^{\text {Alfl }} \mathrm{Tg}^{\text {cD4cre }}$ mice (Supplemental Figure 6A). To determine the effect of Tfh cell depletion on hypertension, we infused Ang II for 4 weeks in $B c l 6^{f l f l} \mathrm{Tg}^{\mathrm{CD} 4 \mathrm{cre}}$ mice and Bcl $6^{l / f l}$ littermate controls. Although the initial rise in SBP was similar in both groups of mice, Tfh cell-deficient mice were unable to sustain the hypertensive response, reaching a SBP approximately $20 \mathrm{mmHg}$ lower than that in floxed control mice by day 28 (Figure 6B). We then assessed aortic smooth muscle cell hypertrophy, mesenteric endothelial function, and vascular inflammation in these mice. Aortic media thickness was significantly decreased in the Tfh cell-deficient mice following Ang II infusion, with no change in endothelium-dependent relaxation (Figure 6, C and D, and Supplemental Figure 6B), similar to that seen in IL-21-deficient mice. In contrast to $I l 21^{-1-}$ mice, there was no change in the overall number of aortic total leukocytes, $\mathrm{CD} 4^{+} \mathrm{T}$ cells, B cells, and Tph cells, but there was a specific reduction in aortic Tfh and GC B cells (Figure 6E). This intermediate phenotype observed in Tfh deficient mice compared with IL-21-deficient mice suggests that there may be additional sources of IL-21, such as Tph cells, that are relevant in hypertension or a compensatory increase in other T cell subsets, such as Th17 cells. Indeed, we isolated splenic T cells and quantified IFN- $\gamma$ and IL-17A production from Tfh cell-deficient and floxed control mice following 4 weeks of Ang II infusion and found no change in IFN- $\gamma$ production, but increased IL-17A production (Supplemental Figure 6C). Taken together, these results highlight the critical role played by Tfh cells in hypertension independent of alterations in other $\mathrm{T}$ cell subsets.

Anti-IL-21 treatment lowers BP and reverses endothelial dysfunction and vascular inflammation. To determine whether pharmacologic targeting of IL-21 may be a beneficial therapeutic strategy, WT mice were infused with 
A
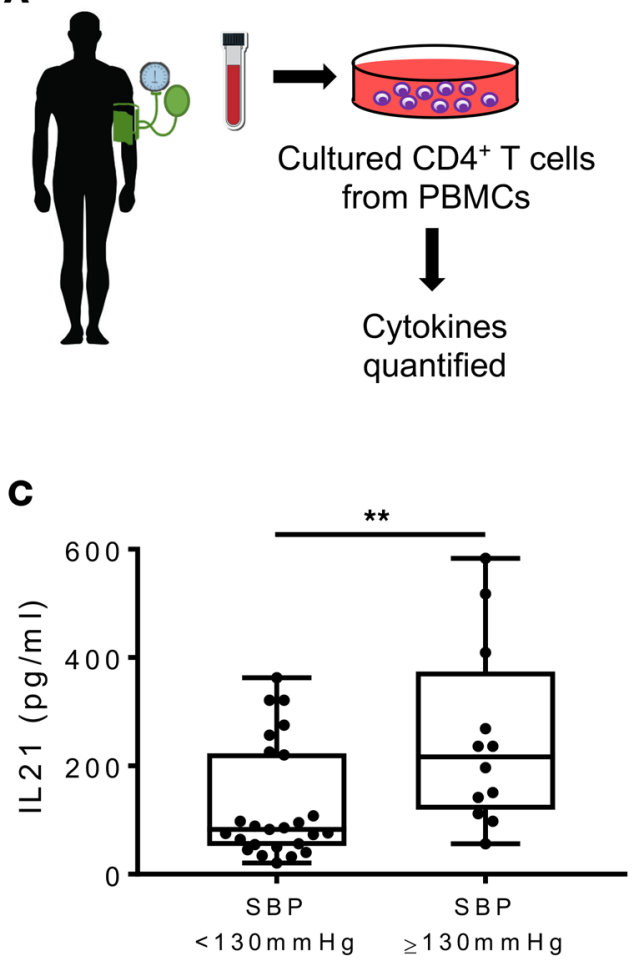

B

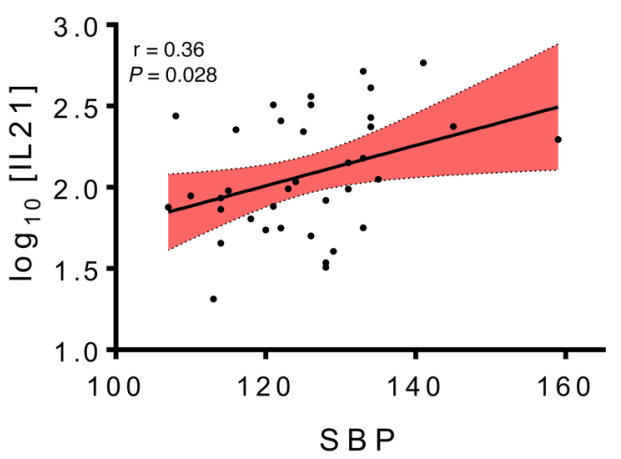

D

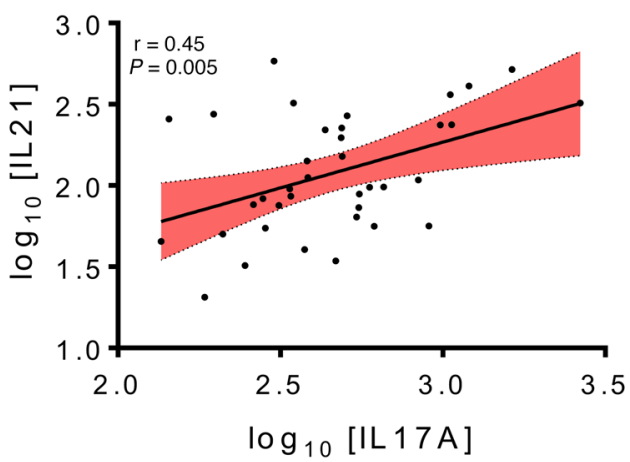

Figure 9. CD4 ${ }^{+}$T cell production of IL-21 correlates with systolic BP and IL-17A in humans. (A) Systolic BP (SBP) was determined at the time of blood draw in human subjects. CD4 ${ }^{+} T$ cells were isolated from PBMCs and cultured for 3 days. Cytokines were then quantified from the supernatants. (B) CD4+ $T$ cell production of IL-21 versus SBP in humans ( $n=37)$. (C) Human CD4 ${ }^{+}$T cell production of IL-21 dichotomized by SBP $<130 \mathrm{mmHg}$ or $\geq 130 \mathrm{mmHg}(n=12-25)$. (D) CD4 T cell production of IL-21 versus IL-17A in humans $(n=37)$. Pearson's correlation coefficient and corresponding $P$ value are shown on the graph (B and $\mathbf{D})$. Data are expressed as box-and-whisker plots (C); ${ }^{* *} P<0.01$ by Student's $t$ test (C).

Ang II for 4 weeks and randomized to receive twice weekly injections of isotype control or an anti-IL-21 neutralizing antibody (100 $\mu \mathrm{g}$ /injection) during the last 2 weeks. Mice that received anti-IL-21 treatment exhibit an approximately $15-\mathrm{mmHg}$ reduction in BP (Figure 7A). Importantly, anti-IL-21 treatment restored endothelium-dependent relaxation to Ach to levels comparable with vehicle-infused WT animals (Figure 7B). Endothelium-independent relaxation to SNP was similar in both groups (Supplemental Figure 7). In addition, anti-IL-21 treatment decreased aortic inflammatory cells, including Tph, Tfh, and GC B cells, to levels comparable to those in vehicle-infused WT animals (Figure 7, C-E). Thus, inhibition of IL-21 lowers BP and reverses endothelial dysfunction and vascular inflammation.

Recombinant IL-21 impairs endothelium-dependent relaxation ex vivo and decreases NO production from cultured human aortic endothelial cells. To determine whether IL-21 has direct vascular effects independent of T and B cells, we isolated third-order mesenteric arterioles from WT mice and assessed endothelium-dependent relaxation to Ach before and after 1 hour of incubation with recombinant IL-21 protein (100 ng/ml) or vehicle (PBS). IL-21 but not vehicle treatment resulted in impaired endothelium-dependent relaxation (Figure 8, A and B). We have previously shown that vascular NO levels are decreased in WT mice in response to Ang II infusion (19). To determine the effect of IL-21 on endothelial NO production, we cultured human aortic endothelial cells (HAECs) with vehicle or recombinant IL-21 and assessed NO production using flow cytometry with the DAF-FM dye. IL-21 decreased NO production from these cells (Figure 8C). Thus, IL-21 has direct detrimental effects on the vessel wall independent of alterations in $\mathrm{T}$ and $\mathrm{B}$ cells.

$C D 4^{+}$T cell production of IL-21 correlates with SBP and IL-17A in humans. To determine the relevance of our findings to human hypertension, we quantified IL-21 and IL-17A production from cultured CD4 ${ }^{+} \mathrm{T}$ cells isolated from the peripheral blood of 37 subjects (Figure 9A and Supplemental Table 1). SBP was measured on the day of blood draw and significantly correlated with IL-21 levels (Figure 9B). In addition, when stratified by SBP less than, greater than, or equal to $130 \mathrm{mmHg}$, IL-21 production from CD4 ${ }^{+}$ 


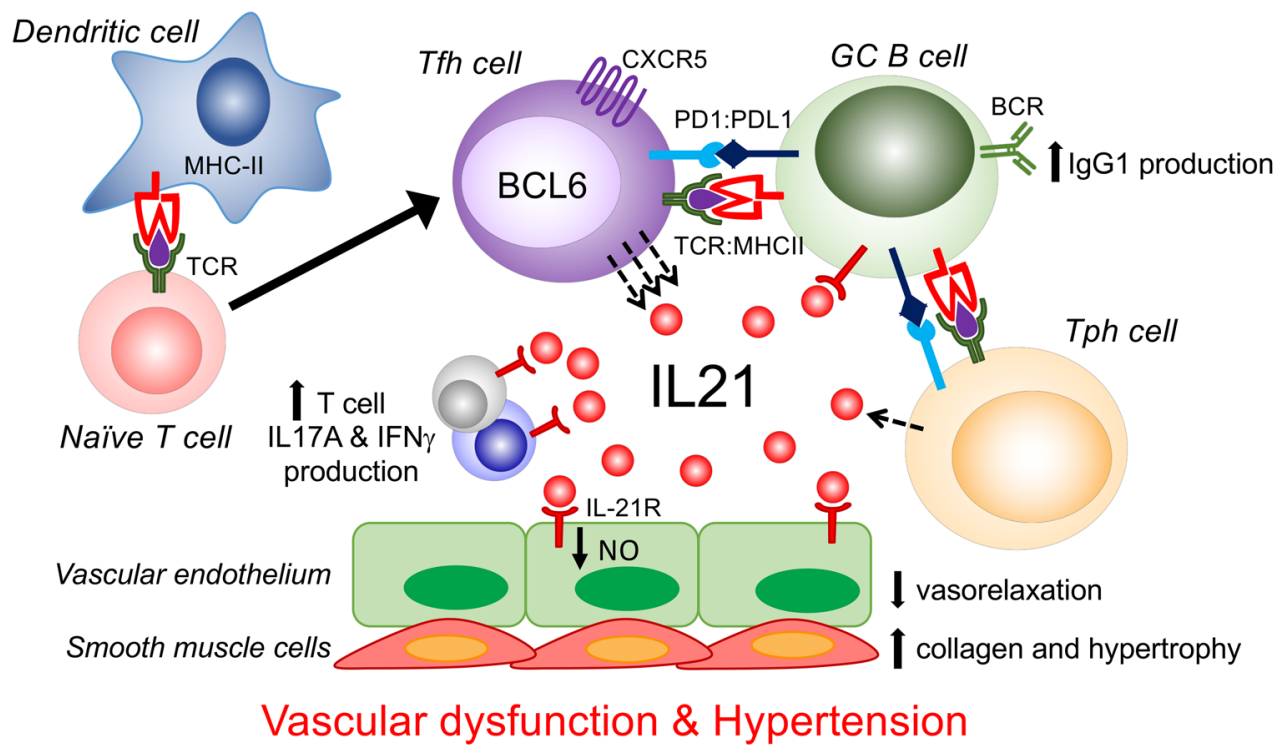

Figure 10. Working model of how IL-21 functions as a master cytokine in hypertension coordinating $\mathrm{T}$, B, and somatic cell responses. TCR, T cell receptor; MHCII, major histocompatibility complex II; BCR, B cell receptor.

T cells was significantly higher in those with SBP greater than or equal to $130 \mathrm{mmHg}$ (Figure 9C). In this sample set, IL-21 levels did not differ significantly between Black and White individuals or between males and females, and did not correlate with BMI or age. Furthermore, T cell production of IL-21 strongly correlated with IL-17A production (Figure 9D), and this correlation remained significant in multivariate analysis after controlling for race, sex, age, and BMI. This finding suggests that the same or similar cells are producing IL-21 and IL-17A in human hypertension and/or that one cytokine strongly regulates the other. As described above, IL-21 is known to promote IL-17A production. Taken together, these data suggest that IL-21 and the cells that produce it are an attractive therapeutic target for hypertension and its vascular complications.

\section{Discussion}

It is now evident that hypertension is an inflammatory disease, with key roles played by most innate and adaptive immune cells, including DCs, monocytes/macrophages, $\gamma \delta \mathrm{T}$ cells, $\mathrm{CD} 4^{+} \mathrm{T}$ helper cells, $\mathrm{CD} 8^{+}$cytotoxic $\mathrm{T}$ cells, and B cells (7). Modest elevations of serum Igs have also been observed in both experimental and human hypertension $(24,25)$, but whether hypertension is associated with a GC reaction is not known. Here we show that genetic or pharmacological inhibition of IL-21 results in blunted hypertension and reduced vascular inflammation/dysfunction. Since IL-21 is produced primarily by Tfh cells whose main effector function is to promote a GC reaction, our data strongly suggest that hypertension is associated with a GC response. Indeed, we show accumulation of GC-like cells in the aorta and mesenteric lymph nodes and increased IgG1 production in hypertensive WT but not $I / 21^{-/-}$mice. Moreover, genetic deficiency of Tfh cells results in protection from chronic hypertension. Finally, we show that $\mathrm{BP}$ in a cohort of humans correlates with $\mathrm{CD} 4^{+} \mathrm{T}$ cell production of IL-21. This latter finding is consistent with a previous report showing that among patients with coronary artery disease, serum IL-21 was upregulated in those with concomitant hypertension compared with those without (26).

IL-21 is a pleiotropic cytokine with effects on innate and adaptive immune cells as well as nonimmune cells $(10,27)$. IL-21 has been shown to promote Th17 and Th1 cells and inhibit Tregs (11, 20). We and others demonstrated that hypertension is associated with an increase in IL-17A and IFN- $\gamma$ production, and that these cytokines play important roles by modulating renal sodium transporters, vascular fibrosis, and endothelial function $(20,28,29)$. Here, we show that loss of IL-21 abrogates hypertension-induced T cell production of IL-17A and IFN- $\gamma$. IL-21 is also the most potent cytokine known to induce GC B cell proliferation and differentiation into antibody-secreting plasma cells (20). In keeping with this, we found that hypertension is associated with increased accumulation of GC-like cells in the vasculature and secondary lymphoid organs in an IL-21-dependent manner. Finally, we show that IL-21 has direct effects on vascular 
endothelial cells, altering bioavailability of NO and promoting endothelial dysfunction. Future studies are needed to determine mechanisms by which IL-21 alters superoxide and NO levels and to investigate whether IL-21 has direct effects on vascular smooth muscle cells and fibroblasts to increase chemokine expression and collagen synthesis. In line with this, it has been shown in inflammatory bowel disease that colonic myofibroblasts and epithelial cells respond to IL-21 by secreting matrix metalloproteases and chemokines that recruit other inflammatory cells (27). These potential effects of IL-21 in hypertension are not mutually exclusive, and thus we propose that IL-21 may indeed function as a "master" cytokine in hypertension, orchestrating responses of T cells, B cells, and parenchymal cells (Figure 10).

The finding that hypertension is associated with a GC reaction has important therapeutic implications. The primary purpose of a GC reaction is to mount a specific and effective immune response in the setting of infection or vaccination. GCs are transient microanatomical sites in secondary or tertiary lymphoid organs where somatic hypermutation (to generate high-affinity antibodies) and isotype class switching (e.g., IgM $\rightarrow \operatorname{IgG}$ ) occurs. After multiple rounds of affinity maturation, B cells exit GCs as memory B cells or long-lived plasma cells. B cells can also differentiate along an extrafollicular pathway and produce IgM or class-switched antibodies with a low degree of somatic mutation. Although autoimmune diseases may use either pathway, there is emerging data that a GC response is the predominant pathway at least in some autoimmune diseases (22, 30-32). Interestingly, Clement et al. recently demonstrated that experimental atherosclerosis is associated with an overactive Tfh/GC B cell axis in secondary lymphoid organs and increased development of tertiary lymphoid organs in the aorta. Moreover, these authors demonstrated the presence of Th cells within tertiary lymphoid organs of human atherosclerotic aneurysmal arteries (33). We found that experimental hypertension is associated with both structured and unstructured $\mathrm{T}$ and $\mathrm{B}$ cell aggregates, increased accumulation of aortic Tfh and GC B cells, and an increased percentage of lymph node GC B cells. To determine the role of Tfh cells in hypertension, we created mice with T cell-specific deletion of the Tfh cell lineage determining transcription factor BCL6. Mice with T cell deletion of BCL6 had blunted accumulation of Tfh cells and GC B cells in the aorta and were unable to sustain the hypertensive response to chronic Ang II infusion, suggesting a critical role for a GC reaction in hypertension.

Of note, mice with T cell deletion of BCL6 exhibited delayed protection from Ang II-induced BP elevation and were not protected from vascular accumulation of other immune cells, in contrast to $I l 21^{-1}$ mice. One explanation for this is that other immune cells such as NKT cells and/or Th17 cells are also important sources of IL-21 in hypertension or are able to compensate in the presence of Tfh cell deficiency. Indeed, we detected an increase in splenic CD4+ $\mathrm{T}$ cell production of IL-17A in the Tfh cell-deficient mice following Ang II infusion. Another explanation is that our model did not deplete the Tfh-like "peripheral helper T cells" or Tph cells, which do not express prototypic Tfh markers such as CXCR5 or BCL6 but still produce factors important in B cell help such as IL-21. Recent studies demonstrate that these Tfh-like cells cooperate with B cells in unstructured aggregates of inflamed tissues and may be important in the local differentiation of B cells into antibody-secreting cells (34). For example, Rao and colleagues used mass cytometry to analyze $\mathrm{T}$ cells isolated from the joints and blood of patients with rheumatoid arthritis. They identified an expanded population of PD- ${ }^{\text {hi }}$ CXCR5 ${ }^{-} \mathrm{CD} 4{ }^{+} \mathrm{T}$ cells, which they named Tph cells, distinct from PD- ${ }^{\text {hi }} \mathrm{CXCR} 5^{+} \mathrm{CD} 4^{+} \mathrm{Tfh}$ cells, and showed that these cells express IL-21 and chemokine receptors such as CCR5 that direct migration to inflamed sites (16). Indeed, when we quantified PD- $1^{\text {hi }} \mathrm{CXCR} 5^{-} \mathrm{CD} 4^{+} \mathrm{T}$ cells in our animal models, we detected an increase in Tph cells in the aorta of WT but not $I l 21^{-1-}$ mice (Figure 4C). In addition, as expected, these cells were not reduced in mice with $\mathrm{T}$ cell deletion of BCL6 (Figure 6E). It is not clear to what extent Tph-B cell interactions in peripheral tissues are similar to classic Tfh-GC B cell interactions in secondary lymphoid organs. Further studies are needed to elucidate the relative role of Tph versus Tfh cells in hypertension.

We and others have elucidated the role of various $\mathrm{T}$ effector and regulatory cell subsets in hypertension pathogenesis (7). Recently Chan et al. demonstrated that mice with genetic or pharmacological depletion of B cells develop blunted Ang II-induced hypertension (9). It has also been shown that DCs are activated in hypertension and present immunogenic isolevuglandin-modified peptides to $\mathrm{T}$ cells that promote $\mathrm{T}$ cell proliferation (23). A missing link has been how these various immune cells produce a coordinated and orchestrated response. Based on our results, we propose that hypertensive DCs induce naive $\mathrm{T}$ cells to differentiate into Tfh cells. Tfh cells then induce a GC reaction in the aorta and secondary lymphoid organs, leading to increased IgG production. IL-21 production from Tfh cells, and perhaps other sources such as Tph and Th17 cells, promotes the production of pathogenic IL-17A- and 
IFN- $\gamma$-producing $\mathrm{T}$ cell subsets and may have direct effects on parenchymal cells, leading to hypertension and end-organ dysfunction (Figure 10). Further studies are necessary to more precisely define the nature and mechanism of action of $\mathrm{B}$ cells and Igs in hypertension.

Our data reveal some subtle differences between $I l 21^{-{ }^{-}}$animals and acute depletion of IL-21 with neutralizing antibodies. Anti-IL-21 antibody treatment appears to be more effective at reducing vascular inflammation. In addition, while endothelium-dependent relaxation in response to Ach is impaired at baseline in $I l 21^{-1-}$ mice, anti-IL-21 antibody treatment restores endothelial function to the levels seen in normotensive WT mice. When we assessed mesenteric arteriolar superoxide production in the IL-21-deficient mice, it was elevated at baseline compared with WT animals. This may explain the baseline impairment in vascular function and suggests that IL-21 may have some protective effects at early developmental stages or at low levels. Furthermore, compensatory changes may have occurred in the mice deficient in IL-21 from birth. Nevertheless, these complementary approaches for deleting IL-21 demonstrate the critical role played by this cytokine in hypertension and end-organ damage and suggest that anti-IL-21 treatment may be a promising therapeutic strategy for hypertension.

Phase I or II clinical trials using monoclonal anti-IL-21 antibodies for lupus, rheumatoid arthritis, and Crohn's disease are either completed or in progress (10). Hypertension is highly prevalent in patients with autoimmune diseases, and thus it would be interesting to determine how this therapy affects BP and vascular dysfunction. However, the risk-to-benefit ratio of such therapeutics should be carefully considered, since IL-21 also has potent antitumor and antiviral activities. In fact, IL-21 is currently being investigated as a therapeutic agent in solid tumors and hematopoietic malignancies. Boosting IL-21 levels may also be beneficial for viral infections such as hepatitis B and C and HIV. Thus, tissue-restricted targeting of a specific cellular source of IL-21, such as Tfh or Tph cells, if feasible, would be a more attractive therapeutic option.

In summary, we show that both experimental and human hypertension is associated with increased $\mathrm{CD}^{+} \mathrm{T}$ cell production of IL-21. Mice deficient in IL-21 exhibit blunted hypertension and vascular end-organ dysfunction. Pharmacological inhibition of IL-21 after the onset of hypertension lowers BP and reverses endothelial dysfunction and vascular inflammation. Furthermore, hypertension is associated with increased aortic Tfh and GC B cells, and Tfh cell deficiency protects from chronic Ang II-induced hypertension. To our knowledge, this is the first study implicating a GC reaction in hypertension and suggests that inhibition of IL-21 or specific depletion of IL-21-producing cells may be a novel therapeutic strategy for the treatment of hypertension and its micro- and macrovascular complications.

\section{Methods}

Animals and experimental hypertension. WT C57BL/6J mice, CD4-Cre transgenic mice ( $\left.\mathrm{Tg}^{\mathrm{CD} 4 \mathrm{cre}}\right)$, and $\mathrm{Bcl}^{\text {fl/fl}}$ mice were purchased from the Jackson Laboratory. $I l 21^{-1-}$ mice were generated as previously described (35). These mice were backcrossed more than 10 generations to C57BL/6J mice. Age- and sex-matched mice between 10 and 12 weeks of age were used. Experimental hypertension was induced using the Ang II model or DOCA-salt treatment. Mice were anesthetized with isoflurane via nose cone or ketamine/xylazine $(90-120 \mathrm{mg} / \mathrm{kg}+10$ $\mathrm{mg} / \mathrm{kg} ; 1: 1$ volume) via intraperitoneal injection. For Ang II-induced hypertension, 28-day (Alzet, DURECT, model 2004) or 14-day (Alzet, DURECT, model 2002) osmotic minipumps containing Ang II (490 ng/kg/min) (MilliporeSigma, catalog A2900) or vehicle ( $0.08 \mathrm{M}$ sodium chloride $+1 \%$ acetic acid solution) were implanted subcutaneously. For DOCA-salt treatment, uninephrectomy was performed, a DOCA pellet (100 mg; Innovative Research of America) was implanted subcutaneously, and the drinking water was supplemented with 1\% $\mathrm{NaCl}$ for 21 days. Animals were euthanized after 7, 14, 21, or 28 days as indicated by $\mathrm{CO}_{2}$ inhalation.

$B P$ measurement. BP was measured twice weekly and averaged using a noninvasive tail-cuff platform (Hatteras Instruments) or invasively by carotid radiotelemetry as previously described (14). Mice were allowed to recover for 10-14 days after telemetry implantation prior to obtaining baseline BPs and implantation of osmotic minipumps.

Murine T cell isolation, culture, and cytokine quantification. Spleen homogenates were filtered through a 40- $\mu \mathrm{m}$ cell strainer, followed by depletion of red blood cells using RBC lysis buffer (eBioscience). CD4 ${ }^{+}$and CD8 ${ }^{+}$ $\mathrm{T}$ cells were isolated from splenic single-cell suspensions using Miltenyi cell separation kits according to the manufacturer's instructions and sorted using an autoMACS magnetic cell sorter (Miltenyi Biotec). Cells were plated in RPMI 1640 media $+10 \%$ FBS $+1 \%$ penicillin/streptomycin $+50 \mu \mathrm{M} \beta$-mercaptoethanol at a density of 200,000 cells $/ 100 \mu$ on a non-tissue culture-treated 96 -well plate coated with mouse anti-CD3 $(2 \mu \mathrm{g} /$ $\mathrm{ml}$ ) and mouse anti-CD28 (2 $\mu \mathrm{g} / \mathrm{ml})$ antibodies (BD Biosciences) for 72 hours. Murine IL-17A, IFN- $\gamma$, IL-10, and IL-21 were measured from cell culture supernatants by ELISA Ready-SET-Go! Kits (eBioscience). 
$R N A$ isolation and $q R T-P C R$. RNA was isolated from cells using an RNeasy Mini Kit (QIAGEN) according to the manufacturer's instructions. Total RNA concentration was measured using a DS-11 spectrophotometer (DeNovix). A High-Capacity cDNA Reverse Transcription Kit (Applied Biosystems) was used according to the manufacturer's instructions to make cDNA. The TaqMan assay system (Thermo Fisher Scientific) was used to detect Il21, Bcl6, and Gapdh (endogenous control). Relative quantification was determined using the comparative CT method, with data normalized to Gapdh and calibrated to the average of the control group.

Immunohistochemistry, histological staining, and imaging. 10\% formalin-fixed, paraffin-embedded tissue sections of aorta or spleen from WT or $I l 21^{-/-}$mice treated for 28 days with Ang II or vehicle were sectioned at 5- $\mu \mathrm{m}$ thickness. Slides were labeled with anti-CD3 (ab16669, Abcam, 1:250 dilution), anti-B220 (553086, BD Pharmingen, 1:20,000 dilution), or PNA (B1075, Vector, 1:250 dilution) to detect T cells, B cells, and GC B cells, respectively. For collagen staining, slides were stained with Masson's trichrome blue or Picrosirius red as previously described $(19,36)$. Slides were scanned at $\times 20$ using a Leica SCN400 Slide Scanner and visualized using Digital Image Hub software (Leica Biosystems).

Vascular reactivity studies. Isometric tension studies were conducted using 2-mm segments of third-order mesenteric arterioles dissected free of perivascular fat. Studies were performed in a small vessel horizontal wire myograph (Danish Myo Technology, models 610M and 620M) as previously described (14). Vessels were preconstricted with norepinephrine prior to treatment with increasing doses of Ach or SNP. For ex vivo IL-21 treatment experiments, vessels were incubated with recombinant murine IL-21 (100 ng/ml; Peprotech) or vehicle (PBS) in the organ chamber for 1 hour between assessments of endothelium-dependent relaxation to Ach.

Mesenteric vessel superoxide measurement. Superoxide levels were measured from mesenteric arterioles by quantification of 2-hydroxyethidium (2-OH Eth) from dihydroethidium (DHE) by high-performance liquid chromatography. The 2-OH Eth product specifically reflects the interaction of DHE with superoxide as previously validated (37).

Fluorescence flow cytometry staining of lymph node and aortic leukocytes. Mesenteric lymph nodes free of surrounding fat were homogenized and filtered through a 40- $\mu \mathrm{m}$ cell strainer. Single-cell suspensions of whole aorta with adjacent perivascular fat were isolated as previously described (38). Samples were stained for viability and surface markers using the following reagents: for the T cell panel, LIVE/DEAD Fixable Violet Dead Cell Stain (Life Technologies), brilliant violet 510 (BV510) anti-CD45 (BioLegend, clone 30-F11), peridinin chlorophyll protein-cyanin-5.5 (PerCp-Cy5.5) anti-CD3 antibody (BioLegend, clone 17A2), allophycocyanin (APC) anti-CD8 antibody (eBioscience, clone 53-6.7), allophycocyanin-cyanin-7 (APC-Cy7) anti-CD4 antibody (BioLegend, clone GK1.5), phycoerythrin-cyanin-7 (PE-Cy7) anti-CXCR5 antibody (BD Pharmingen, clone 2G8), and FITC anti-PD-1 (eBioscience, clone J43); for the B cell panel: LIVE/DEAD Fixable Violet Dead Cell Stain (Life Technologies), BV510 anti-CD45 (BioLegend, clone 30-F11), PerCp-Cy5.5 anti-CD45R/ B220 antibody (BD Biosciences, clone RA3-6B2), APC anti-IgD antibody (eBioscience, clone 11-26), APCCy7 anti-CD138 antibody (BioLegend, clone 281-2), PE-Cy7 anti-CD19 antibody (BD Pharmingen, clone 1D3), PE anti- GL7 (Invitrogen, clone GL-7), FITC anti-Fas (eBioscience, clone 15A7); for the innate immune cell panel: LIVE/DEAD Fixable Violet Dead Cell Stain (Life Technologies), BV510 anti-CD11b (BioLegend, clone M1/70), PerCp-Cy5.5 anti-CD3 antibody (BioLegend, clone 17A2), APC anti-NK1.1 antibody (BioLegend, clone PK136), APC-Cy7 anti-F4/80 antibody (BioLegend, clone BM8), PE anti-Ly6G (BioLegend, clone 1A8), and FITC anti-CD45 (BioLegend, clone 30-F11). Staining was performed in $100 \mu 1$. All antibodies were used at 1:100 dilution. Samples were acquired on a BD FACSCanto II system and analyzed using CytoBank. Gates were applied using FMO controls. A known quantity of counting beads (123count eBeads, eBioscience) was added to each sample prior to acquisition. Results were normalized using the bead count.

Antibody treatments. Mice were implanted with 28-day osmotic minipumps containing Ang II. After 14 days of Ang II infusion, mice were randomized to either an isotype control group (eBioscience, rat IgG2 k isotype control, functional grade purified, clone eBRa) or anti-mouse IL-21 treatment group (eBioscience, anti-mouse IL-21 functional grade purified, clone FFA21). Treatment was administered by intraperitoneal injection with $100 \mu \mathrm{g}$ antibody twice a week for the last 2 weeks of Ang II infusion (days 16, 19, 22, and 25). This regimen was chosen based on prior studies (39).

Plasma Ig isolation and quantification. Mice were euthanized, and whole blood was collected by direct cardiac puncture into an EDTA-coated syringe. Blood was immediately centrifuged and plasma stored at $-80^{\circ} \mathrm{C}$. Plasma Ig was quantified using a LEGENDplex Mouse Immunoglobulin Isotyping Panel (BioLegend) according to the manufacturer's instructions. 
$D C-C D 4^{+} T$ cell coculture. Splenic single-cell suspensions were generated as described above. DCs $\left(\mathrm{CD} 11 \mathrm{c}^{+}\right)$and naive $\mathrm{CD} 4^{+} \mathrm{T}$ cells $\left(\mathrm{CD} 44^{-}\right)$were enriched using Miltenyi cell separation kits (catalog 130 108-338 and 130-104-453) according to the manufacturer's instructions and sorted using an autoMACS magnetic cell sorter (Miltenyi Biotec). 200,000 cells were plated in duplicate at a 1:5 ratio (DC/T cells) in RPMI 1640 media $+10 \%$ FBS $+1 \%$ penicillin/streptomycin $+50 \mu \mathrm{M} \beta$-mercaptoethanol $+1 \mathrm{mM}$ sodium pyruvate $+1 \%$ L-glutamine at a density of $1 \times 10^{6} \mathrm{cells} / \mathrm{ml}$ in a tissue culture-treated 96 -well round-bottom plate for 72 hours.

NO measurement from cultured endothelial cells. HAECs were purchased from Lonza (CC-2535, lot 0000297640) and grown to 90\% confluency in endothelial cell growth medium (PromoCell). The cells were detached using trypsin, resuspended in endothelial cell media, and allowed to rest for 30 minutes at $37^{\circ} \mathrm{C}$. Cells were loaded for 30 minutes with $1 \mu \mathrm{M}$ 4-amino-5-methylamino-2', $7^{\prime}$-diflurofluorescein diacetate (DAF-FM diacetate; Thermo Fisher Scientific) dissolved in DMSO. Cells were then incubated with $100 \mathrm{ng} / \mathrm{ml}$ recombinant human IL-21 (PeproTech) or vehicle (PBS). After 15 minutes, the cells were acquired via flow cytometry. Gating and data analysis were performed in FlowJo.

Human subjects. Blood samples were obtained as part of Vanderbilt University's American Heart Association Strategically Focused Research Network protocol. Inclusion criteria were age 30-80 years, SBP 110-150 mmHg, and/or diastolic BP 80-99 mmHg on the day of the study visit. BP was measured in the seated position after a 10- to 15-minute rest period by an automated cuff (GE Critikon Dinamap Pro 1000). Exclusion criteria were pregnancy, intolerance to study protocols, acute cardiovascular events within the previous 6 months, impaired renal function (estimated glomerular filtration rate [GFR] $<45$ $\mathrm{ml} / \mathrm{min} / 1.73 \mathrm{~m}^{2}$ ), current or recent (within $1 \mathrm{month}$ ) treatment with systemic glucocorticoid therapy, current use of hypertensive drugs (except calcium channel blockers and beta blockers), pharmacologically treated diabetes mellitus, morbid obesity (BMI >45), prior adverse reaction to thiazide or spironolactone, contraindications to MRI, impaired hepatic function (aspartate amino transaminase and/or alanine amino transaminase $>1.5$ times the upper limit of normal range), current illicit drug use, and nonuse of birth control by sexually active women of childbearing potential.

Human T cell isolation, culture, and cytokine quantification. PBMCs were isolated from whole blood by Ficoll plaque centrifugation (GE Life Science). $\mathrm{CD}^{+} \mathrm{T}$ cells were isolated from the Ficoll buffy coat using Miltenyi cell separation kits according to the manufacturer's instructions and sorted using an autoMACS magnetic cell sorter (Miltenyi Biotec). Cells were plated in RPMI 1640 media $+10 \% \mathrm{FBS}+1 \%$ penicillin/ streptomycin $+50 \mu \mathrm{M} \beta$-mercaptoethanol at a density of 200,000 cells $/ 200 \mu 1$ on a non-tissue culturetreated 96-well plate coated with human anti-CD3 $(2 \mu \mathrm{g} / \mathrm{ml})$ and human anti-CD28 $(2 \mu \mathrm{g} / \mathrm{ml})$ antibodies (BD Biosciences) for 72 hours. Human IL-21 and IL-17A were measured from cell culture supernatants by LEGENDplex bead assay (BioLegend).

Statistics. All data are expressed as mean \pm SEM or as box-and-whisker plots, with the bounds of the boxes representing the 25 th to 75 th percentiles, the line within the box representing the median, and the whiskers representing the minimum to maximum values. Data were analyzed in GraphPad Prism or $\mathrm{R}$ using Student's $t$ test (for 2-group comparisons) or 2-way ANOVA (for multiple-group comparisons). Nonparametric 2-group data were analyzed by Mann Whitney $U$ test. Vascular relaxation studies were compared using area-under-the-curve analysis. Human cytokine data were log transformed for Pearson's correlation analysis. $P<0.05$ was considered significant.

Study approval. All animal procedures were approved by the Vanderbilt IACUC. The human studies were approved by the Vanderbilt Institutional Review Board (IRB\#141382) and conform to the standards set forth by the US Federal Policy for the Protection of Human Subjects. All human subjects provided written informed consent prior to inclusion in the study.

\section{Author contributions}

BLD and MSM designed the experiments. BLD, AKP, YC, CDS, FL, MA, LX, HAI, AED, SID, AEN, HMSA, and MRA generated data. BLD, AKP, MA, AED, SZ, and MSM analyzed data. MSM supervised the study. JDF, NRB, JPV, SMD, and AA generated human data. BLD, FE, and MSM analyzed human data. TAI and MSM supervised the human study. BLD, AKP, FL, NRB, FE, JPV, and MSM wrote the manuscript and revised it for intellectual content. 


\section{Acknowledgments}

We acknowledge the Vanderbilt Translational Pathology Shared Resource (NIH Cancer Center 5P30 CA68485-19), Digital Histology Shared Resource at VUMC, and the Vanderbilt Free Radicals in Medicine Core (FRIMCORE) (NIH P01 HL129941). This work was supported by an NIH T32 training grant (T32 HL069765-11A1) and an American Heart Association (AHA) predoctoral fellowship award (17PRE33460032) to BLD; an NIH T32 training grant (T32 HL7411-37) to AKP; an AHA Strategically Focused Prevention Research Network award to TAI and MSM; a Veterans Affairs Merit Review award (IBX000915A) and a pilot grant through VUMC's Digestive Disease Research Center supported by NIH grant P30DK058404 (to HMSA); and an NIH K08 award (HL121671) and an NIH DP2 award (HL137166) to MSM.

Address correspondence to: Meena S. Madhur, Department of Medicine, Division of Clinical Pharmacology, 2215 Garland Avenue, P415D MRB IV, Vanderbilt University, Nashville, Tennessee 37232, USA. Phone: 615.875.3273; Email: meena.madhur@vanderbilt.edu.

AKP's present address is: Division of Cardiovascular Medicine, Brigham and Women's Hospital, Harvard Medical School, Boston, Massachusetts, USA.

HAI's present address is: Department of Pharmacology and Toxicology, American University of Beirut, Beirut, Lebanon.

AEN's present address is: Division of Allergy, Pulmonary, and Critical Care, VUMC, Nashville, Tennessee, USA.

1. Whelton PK, et al. 2017 ACC/AHA/AAPA/ABC/ACPM/AGS/APhA/ASH/ASPC/NMA/PCNA Guideline for the prevention, detection, evaluation, and management of high BP in adults: executive summary: a report of the American College of Cardiology/American Heart Association Task Force on Clinical Practice Guidelines. Hypertension. 2018;71(6):1269-1324.

2. GBD 2015 Risk Factors Collaborators. Global, regional, and national comparative risk assessment of 79 behavioural, environmental and occupational, and metabolic risks or clusters of risks, 1990-2015: a systematic analysis for the Global Burden of Disease Study 2015. Lancet. 2016;388(10053):1659-1724.

3. Nwankwo T, Yoon SS, Burt V, Gu Q. Hypertension among adults in the United States: National Health and Nutrition Examination Survey, 2011-2012. NCHS Data Brief. 2013;null(133):1-8.

4. Okuda T, Grollman A. Passive transfer of autoimmune induced hypertension in the rat by lymph node cells. Tex Rep Biol Med. 1967;25(2):257-264.

5. White FN, Grollman A. Autoimmune factors associated with infarction of the kidney. Nephron. 1964;1:93-102.

6. Guzik TJ, et al. Role of the T cell in the genesis of angiotensin II induced hypertension and vascular dysfunction. $J$ Exp Med. 2007;204(10):2449-2460.

7. Norlander AE, Madhur MS, Harrison DG. The immunology of hypertension. J Exp Med. 2018;215(1):21-33.

8. Norlander AE, Madhur MS. Inflammatory cytokines regulate renal sodium transporters: how, where, and why? Am J Physiol Renal Physiol. 2017;313(2):F141-F144.

9. Chan CT, et al. Obligatory role for B cells in the development of angiotensin II-dependent hypertension. Hypertension. 2015;66(5):1023-1033.

10. Spolski R, Leonard WJ. Interleukin-21: a double-edged sword with therapeutic potential. Nat Rev Drug Discov. 2014;13(5):379-395.

11. Carbo A, et al. Systems modeling of the role of interleukin-21 in the maintenance of effector CD4+ T cell responses during chronic Helicobacter pylori infection. MBio. 2014;5(4):e01243-e01214.

12. Linterman MA, et al. IL-21 acts directly on B cells to regulate Bcl-6 expression and germinal center responses. $J$ Exp Med. 2010;207(2):353-363.

13. Ozaki K, et al. A critical role for IL-21 in regulating immunoglobulin production. Science. 2002;298(5598):1630-1634.

14. Norlander AE, et al. A salt-sensing kinase in T lymphocytes, SGK1, drives hypertension and hypertensive end-organ damage. JCI Insight. 2017;2(13):null.

15. Magliozzi R, et al. Meningeal B-cell follicles in secondary progressive multiple sclerosis associate with early onset of disease and severe cortical pathology. Brain. 2007;130(Pt 4):1089-1104.

16. Rao DA, et al. Pathologically expanded peripheral T helper cell subset drives B cells in rheumatoid arthritis. Nature. 2017;542(7639):110-114.

17. Chang A, et al. In situ B cell-mediated immune responses and tubulointerstitial inflammation in human lupus nephritis. $J$ Immunol. 2011;186(3):1849-1860.

18. Madhur MS, et al. Interleukin 17 promotes angiotensin II-induced hypertension and vascular dysfunction. Hypertension. 2010;55(2):500-507.

19. Saleh MA, et al. Lymphocyte adaptor protein LNK deficiency exacerbates hypertension and end-organ inflammation. J Clin Invest. 2015;125(3):1189-1202.

20. Lei L, Zhong XN, He ZY, Zhao C, Sun XJ. IL-21 induction of CD4+ T cell differentiation into Th17 cells contributes to bleomycin-induced fibrosis in mice. Cell Biol Int. 2015;39(4):388-399.

21. Mesin L, Ersching J, Victora GD. Germinal center B cell dynamics. Immunity. 2016;45(3):471-482. 
22. DeFranco AL. Germinal centers and autoimmune disease in humans and mice. Immunol Cell Biol. 2016;94(10):918-924.

23. Kirabo A, et al. DC isoketal-modified proteins activate T cells and promote hypertension. J Clin Invest. 2014;124(10):4642-4656.

24. Chan CT, et al. Antibodies in the pathogenesis of hypertension. Biomed Res Int. 2014;2014:504045.

25. Wang X, et al. Relationship of serum immunoglobulin levels to blood pressure and hypertension in an adult population. $J$ Hum Hypertens. 2018;32(3):212-218.

26. Ding R, et al. Effect of serum interleukin 21 on the development of coronary artery disease. APMIS. 2014;122(9):842-847.

27. Liu SM, King C. IL-21-producing Th cells in immunity and autoimmunity. J Immunol. 2013;191(7):3501-3506.

28. Norlander AE, et al. Interleukin-17A regulates renal sodium transporters and renal injury in angiotensin II-induced hypertension. Hypertension. 2016;68(1):167-174.

29. Wu J, et al. Immune activation caused by vascular oxidation promotes fibrosis and hypertension. J Clin Invest. 2016;126(1):50-67.

30. Pissani F, Streeck H. Emerging concepts on T follicular helper cell dynamics in HIV infection. Trends Immunol. 2014;35(6):278-286.

31. Nutt SL, Hodgkin PD, Tarlinton DM, Corcoran LM. The generation of antibody-secreting plasma cells. Nat Rev Immunol. 2015;15(3):160-171.

32. De Silva NS, Klein U. Dynamics of B cells in germinal centres. Nat Rev Immunol. 2015;15(3):137-148.

33. Clement $\mathrm{M}$, et al. Control of the $\mathrm{T}$ follicular helper-germinal center B-cell axis by CD8(+) regulatory $\mathrm{T}$ cells limits atherosclerosis and tertiary lymphoid organ development. Circulation. 2015;131(6):560-570.

34. Hutloff A. T Follicular helper-like cells in inflamed non-lymphoid tissues. Front Immunol. 2018;9:1707.

35. Yi JS, Du M, Zajac AJ. A vital role for interleukin-21 in the control of a chronic viral infection. Science. 2009;324(5934):1572-1576

36. Laroumanie F, et al. LNK deficiency promotes acute aortic dissection and rupture. JCI Insight. 2018;3(20):122558.

37. Fink B, Laude K, McCann L, Doughan A, Harrison DG, Dikalov S. Detection of intracellular superoxide formation in endothelial cells and intact tissues using dihydroethidium and an HPLC-based assay. Am J Physiol, Cell Physiol. 2004;287(4):C895-C902.

38. Laroumanie F, Dale BL, Saleh MA, Madhur MS. Intracellular staining and flow cytometry to identify lymphocyte subsets within murine aorta, kidney and lymph nodes in a model of hypertension. J Vis Exp. 2017;(119):e55266.

39. Saleh MA, Norlander AE, Madhur MS. Inhibition of interleukin 17-A but not interleukin-17F signaling lowers blood pressure and reduces end-organ inflammation in angiotensin II-induced hypertension. JACC Basic Transl Sci. 2016;1(7):606-616. 